\title{
IL DANNO DA "PRODOTTO CONFORME": LE SOLUZIONI EUROPEE E STATUNITENSI NELLA PROSPETTIVA DEL TRANSATLANTIC TRADE AND INVESTMENT PARTNERSHIP (T.T.I.P.)
}

\author{
Enrico Al Mureden \\ Professore Assistente dell'Università di Bologna. \\ enrico.almureden@unibo.it
}

RIASSUNTO: Nel contesto della responsabilità civile degli rapporti di consumo, è analizzata l'armonizzazione di parametri giuridici di responsabilità per "prodotto conforme" ma dannoso tra i sistemi europei e americani e, in vista del Transatlantic Trade and Investment Partnership (TTIP), si propone un'interpretazione unitaria delle norme applicabili. Pertanto, gli standard legali in materia di sicurezza dei prodotti e responsabilità del fabbricante nell'Unione europea e gli Stati Uniti, la distinzione tra prodotto difettoso e il "prodotto conforme" ma dannoso sono esaminati. I risultati hanno confermato la necessità di uniformizzazione delle regole nell'Unione europea a definire un prodotto "ragionevolmente sicuro", così come la responsabilità del produttore secondo la decisione del legislatore sul livello minimo di stabilimento di sicurezza o sul limiti massimi, nel qual caso non c'è responsabilità.

PAROLE CHIAVE: Diritti dei consumatori; Responsabilità del produttore; Prodotto conforme; Transatlantic Trade and Investment Partnership.

O dano do "produto conforme": as soluções europeias e estadunidenses na perspectiva do Acordo de Parceria Transatlântica de Comércio e Investimento (ATP)

RESUMO: No contexto da responsabilidade civil das relações de consumo, analisa-se a harmonização dos parâmetros de responsabilidade por produto conforme, mas danoso, entre os sistemas europeu e americano e, na perspectiva do Acordo de Parceria Transatlântica de Comércio e Investimento (ATP), propõe-se uma interpretação unificada das regras aplicáveis. Para tanto, são examinados os padrões jurídicos relativos à segurança do produto e à responsabilidade do fabricante na União Europeia e nos Estados Unidos, a distinção entre produto defeituoso e o produto conforme, porém danoso. Conclui-se pela necessidade de uniformização de regras nos países da União Europeia para definição de um produto "razoavelmente seguro", assim como para a responsabilidade do fabricante segundo a opção do legislador pelo estabelecimento de nível mínimo de segurança, ou de um limite máximo, caso em que não haverá responsabilidade.

PALAVRAS-CHAVE: Direito do consumidor; Responsabilidade do fabricante; Produto conforme; Acordo de Parceria Transatlântica de Comércio e Investimento.

The damage from "compliant product": European and US solutions from the perspective of the Transatlantic Trade and Investment Partnership (TTIP)

ABSTRACT: In the context of the liability of consumer relations, it analyzes the harmonization of legal parameters of liability for harmful, "compliant product" among the European and

Recebido em: 1 set. 2016. Avaliado em: 22 nov. 2016.

R. Jur. FA7, Fortaleza, v. 13, n. 2, p. 165-189, jul./dez. 2016 
Il danno da "prodotto conforme": le soluzioni europee e statunitensi nella prospettiva del Transatlantic Trade and Investment Partnership (T.T.I.P.)

American systems, and, in view of the Transatlantic Trade and Investment Partnership (TTIP), proposes a unified interpretation of the applicable rules. To this end, the legal standards regarding product safety and manufacturer liability in the European Union and the United States, and the distinction between the defective product and the harmful, "compliant product" are examined. The results confirm the necessity of standardization of rules in the European Union to define a product "reasonably safe", as well as the responsibility of the manufacturer according to the decision of the legislature on the minimum level of security establishment or the maximum limits, in which case there will be no liability.

KEYWORDS: Consumer Law; Manufacturer liability; Compliant product; Transatlantic Trade and Investment Partnership.

\section{LA SICUREZZA DEI PRODOTTI E LA RESPONSABILITÀ DEL FABBRICANTE NELLA PROSPETTIVA DEL TRANSATLANTIC TRADE AND INVESTMENT PARTNERSHIP (T.T.I.P.)}

A trent'anni dall'adozione della dir. 85/374/CEE, che ha introdotto nei Paesi dell'Unione Europea una disciplina armonizzata della responsabilità del produttore, e a trentadue dall'avvio del c.d. "nuovo approccio"1 (dir. 83/189/CEE), mediante il quale è stato avviato quel processo di armonizzazione degli standards di sicurezza attualmente oggetto di un processo di un'ulteriore implementazione e razionalizzazione (New Legislative Framework) di cui dà ampio conto The Blu Guide on the Implementation of EU Product Rules, pubblicata dalla Commissione Europea nel 2014, il tema della sicurezza dei prodotti e della responsabilità del fabbricante non sembrano aver conosciuto negli ordinamenti europei e, in particolare in quello italiano, quella rilevanza che, da ormai cinque decenni caratterizza l'esperienza giuridica nordamericana ${ }^{2}$. Cionondimeno, l'imminente conclusione del Transatlantic Trade and Investment Partnership (T.T.I.P.) o Partenariato Transatlantico per il commercio e gli investimenti ${ }^{3}$, mediante il quale Unione Europea e Stati Uniti mirano ad armonizzare i reciproci standards di sicurezza dei prodotti e a creare uno spazio economico comune, impone all'interprete l'esigenza di una rinnovata attenzione nei riguardi di articolati sistemi di norme (quello sulla responsabilità del fabbricante e quello che può sinteticamente indicarsi con il termine di legislazione sulla sicurezza dei prodotti) tra loro profondamente interconnessi ${ }^{4}$ e destinati ad essere interessati da modificazioni riconducibili in via diretta o indiretta proprio dalla annunciata soppressione delle cosiddette barriere non tariffarie, ossia di quelle disomogeneità ed incongruenze che tuttora caratterizzano i diversi standards di sicurezza dei prodotti negli ordinamenti.

\footnotetext{
1 La Commissione nella sua comunicazione «Armonizzazione tecnica e normalizzazione: un nuovo approccio» ha proposto una revisione dei metodi e delle procedure secondo un nuovo approccio in materia di armonizzazione tecnica e di utilizzazione della normalizzazione. In definitiva l'attuazione dell'armonizzazione tecnica - perseguita in un primo momento mediante direttive specifiche molto tecniche e dettagliate, prodotto per prodotto avrebbe dovuto essere realizzata attraverso disposizioni regolamentari generali applicabili a settori o famiglie di prodotti nonché a tipi di rischio. Sulla base di questa proposta della Commissione e delle sue conclusioni in materia di normalizzazione del 16 luglio 1984 il Consiglio adottò la Risoluzione 7 maggio 1985, relativa ad una nuova strategia in materia di armonizzazione tecnica e normalizzazione (Risoluzione del Consiglio 85/C 136/01, G.U. C.e., C 136, 4 giugno 1985) con la quale ha preso avvio il c.d. nuovo approccio. Sul punto si veda Carnevali, Prevenzione e risarcimento nelle direttive comunitarie sulla sicurezza dei prodotti, in Resp. civ. e prev., 2005 , p. 5.

2 Ponzanelli, Introduzione, R. Pardolesi e Ponzanelli (a cura di), “I 25 anni di products liability”, in Danno e resp., 2012, p. 5.

3 Per una diffusa illustrazione si rinvia al sito ufficiale della Comm. http://ec.europa.eu/trade/policy/in-focus/ttip/.

4 V. The Blu Guide on the Implementation of EU Product Rules, pubblicata dalla Comm. nel 2014, p. 12.
} 
In quest'ottica l'analisi comparatistica del sistema della sicurezza dei prodotti e della responsabilità del fabbricante nell'Unione Europea e negli Stati Uniti assume una valenza che va ben oltre la finalità di individuare elementi di differenziazione e punti di contatto; essa, nella prospettiva di una uniformazione degli standards di sicurezza richiesti ai fini dell'immissione in commercio dei prodotti in uno spazio economico comune, appare un imprescindibile presupposto per l'individuazione di questioni già da tempo emerse nell'ordinamento statunitense e non ancora compiutamente delineate nel nostro sistema legislativo e giurisprudenziale. Tra queste, merita sicuramente particolare attenzione quella della responsabilità del fabbricante per i danni cagionati da un prodotto conforme a standards di sicurezza legislativi. La sua soluzione, infatti, impone all'interprete una lettura sistematica e coordinata delle regole che governano la responsabilità civile del fabbricante e di quelle che definiscono i requisiti di sicurezza dei prodotti, adottando un approccio consolidato nell'esperienza giuridica statunitense e reclamato con crescente intensità dal legislatore dell'Unione Europea. L'imminente conclusione del Transatlantic Trade and Investment Partnership (T.T.I.P.) suggerisce, inoltre, di allargare l'ambito di osservazione ad una prospettiva ancor più ampia e complessa di quella che abbracciava il solo sistema giuridico dell'Unione Europea; una prospettiva nella quale diviene imprescindibile l'esigenza di dare vita ad un sistema di regole uniformi che consenta di superare la frammentazione statale delle discipline e fornire agli operatori economici un quadro normativo di riferimento unitario.

\section{ORIGINI, MOTIVI E CARATTERI DEL "SISTEMA" DELLA SICUREZZA DEI PRODOTTI E DELLA RESPONSABILITÀ DEL FABBRICANTE}

Il termine responsabilità del produttore evoca l'idea di un obbligo risarcitorio che scaturisce da un danno provocato a causa dell'utilizzo di un prodotto che presenti anomalie rispetto ad un ideale modello conforme alla regola dell'arte. Per lungo tempo questo genere di situazioni venivano ricondotte all'applicazione delle norme generali in materia di responsabilità civile ${ }^{5}$; la

\footnotetext{
5 Come noto, il problema della responsabilità del produttore nel nostro ordinamento è stato profondamente indagato già a partire dagli anni Settanta. Con riferimento al periodo precedente l'entra in vigore del d.p.r. 224/1988 si segnalano i contributi di Trimarchi, Rischio e responsabilità oggettiva, Napoli, 1961; Ghidini, La responsabilità del produttore di beni di consumo, Milano, 1970, p. 82; Carnevali, La responsabilità del produttore, Milano, 1974; Alpa, Responsabilità dell'impresa e tutela del consumatore, Milano, 1975; Id., Tutela del consumatore e controlli sull'impresa, Bologna, 1977; Castronovo, Problema e sistema nel danno da prodotti, Milano, 1979. Tra i numerosi contributi successivi all'entrata in vigore del d.p.r. 224/1988 si vedano Salvi, Responsabilità extracontrattuale, XXIX, Milano, 1988, p. 1229; Aa.Vv., Il danno da prodotti in Italia, Austria, Repubblica Federale di Germania, Svizzera a cura di Patti, Padova, 1990; Gorassini, Contributo per un sistema della responsabilità del produttore, Milano, 1990; Alpa, Carnevali, Di Giovanni, Ghidini, Ruffolo, Verardi, La responsabilità per danno da prodotti difettosi, Milano, 1990; Ponzanelli, Comm. alla responsabilità da prodotti difettosi a cura di R. Pardolesi e Ponzanelli, in Nuove leggi civ. e comm., 1989, 3, p. 509; Id., Responsabilità del produttore, in Riv. dir. civ., 1995, II, p. 215 ss.; Id., La responsabilità civile. Profili di diritto comparato, Bologna, 1992, p. 112; Alpa, Bin, Cendon, La responsabilità del produttore, in Tratt. dir. comm. e dir. pubbl. econ. diretto da Galgano, XIII, Padova, 1989; Castronovo, voce Danno da prodotti (dir. it. e straniero), in Enc. giur., X, 1995, 11; Id., voce Responsabilità oggettiva (dir. it. e straniero) in Enc. giur., XXVII, 1991, 11; Franzoni, Dieci anni di responsabilità del produttore, in Danno e resp., 1998, p. 823; Monateri, Illecito e responsabilità civile, in Tratt. dir. priv. diretto da Bessone, Torino, 2002, X, 2, p. 257; Cabella Pisu, Cittadini e consumatori nel diritto dell'Unione Europea, in Contratto e impr. Europa, 2007, II, p. 680; Ead., Ombre e luci nella responsabilità del produttore, in Contratto e impr. Europa, 2008, p. 617 ss; Carnevali, "Produttore" e responsabilità per danno da prodotto difettoso nel codice del consumo, in Resp. civ. e prev., 2009, pp. 1938-1945; Cordiano, sub art. 114, in Codice del consumo annotato con la dottrina e la giurisprudenza a cura di Capobianco, Perlingieri, Napoli, 2009, p. 645; Cafrì, sub art. 114, in Codice del consumo e norme collegate a cura di Cuffaro, III ed., Milano, 2012, p. 603 ss; Franzoni, L'illecito, in Tratt. responsabilità civ., Milano, 2010, II ed., I, p. 661 ss.; Thiene, sub art. 114 c.cons., in
} 
Il danno da "prodotto conforme": le soluzioni europee e statunitensi nella prospettiva del Transatlantic Trade and Investment Partnership (T.T.I.P.)

necessità di concepire un sistema di regole specificamente conformate in funzione delle peculiarità che caratterizzano il rapporto tra coloro che sono danneggiati da un prodotto ed il suo fabbricante costituisce il riflesso delle profonde trasformazioni che hanno interessato la progettazione, la fabbricazione e persino la commercializzazione dei prodotti a partire dall'inizio degli anni Sessanta, determinando in quasi tutti i settori merceologici un passaggio dalla dimensione individuale ed artigianale della produzione a quello della fabbricazione su larga scala, in serie e secondo procedimenti standardizzati ${ }^{6}$. Nel panorama attuale, infatti, è possibile individuare categorie di prodotti che scaturiscono necessariamente da un processo di progettazione, fabbricazione e commercializzazione su larga scala organizzato secondo modalità standardizzate, come, ad esempio, le automobili, i farmaci, i componenti elettronici ed i telefoni cellulari. Per altre tipologie di prodotti, invece, continuano a coesistere processi di fabbricazione artigianali accanto a quelli di fabbricazione su larga scala e standardizzati, che, in ogni caso, assumono una rilevanza preminente. È il caso, ad esempio, degli alimenti, degli indumenti, dei complementi di arredamento o di alcune tipologie di giocattoli, che, sebbene fabbricati in prevalenza mediante modalità industriali e standardizzate, continuano in parte a scaturire da attività individuali ed artigianali.

In prima approssimazione sembra possibile affermare che l'idea di concepire regole particolari che governano il risarcimento dei danni provocati dall'utilizzo di un prodotto costituiscano espressione di un'esigenza che matura necessariamente in un contesto nel quale progettazione, fabbricazione e commercializzazione di massa hanno trasformato profondamente il rapporto che l'utilizzatore instaura con il prodotto e con il suo fabbricante o con colui che lo immette sul mercato. Progettazione, fabbricazione e distribuzione in serie di un prodotto fanno sì che anche la presenza di un'anomalia assuma a sua volta carattere seriale e di larga scala conseguendo una diffusione proporzionale a quella del prodotto stesso. In altri termini, quel difetto di progettazione, fabbricazione ed informazione che in un sistema di produzione artigianale ed individuale era destinato a rimanere circoscritto alla dimensione limitata dei rapporti che il singolo fabbricante avesse instaurato con gli utilizzatori, appare oggi enormemente amplificato laddove la produzione di massa determina l'immissione in commercio di ingenti quantità di prodotti tutti dotati di analoghe caratteristiche e destinati ad una platea di utilizzatori assai vasta.

Proprio questo mutato contesto di fatto è alla base di quella istanza di modificazione del sistema giuridico che ha imposto di sottrarre il rapporto tra produttore e utilizzatore (o danneggiato) alle regole comuni che governano la responsabilità civile per disciplinarlo secondo regole speciali che tengano conto delle peculiarità che caratterizzano questo rapporto. Così, sia nell'Unione Europea, sia negli Stati Uniti, la responsabilità del produttore è stata regolata secondo modalità che hanno condotto all'abbandono della responsabilità per colpa in favore di sistemi di responsabilità oggettiva: la dir. 85/374/CEE sancisce la regola secondo cui la responsabilità del produttore si fonda sulla dimostrazione dell'esistenza di un nesso causale tra il danno subito e l'utilizzo del prodotto (artt.1e 4) ${ }^{7}$; in termini analoghi si esprimono sia la section $402 \mathrm{~A}$

Comm. breve al Diritto dei consumatori diretto da De Cristofaro e Zaccaria, Padova, 2013, p. 732; Albanese, La sicurezza generale dei prodotti e la responsabilità del produttore nel diritto italiano ed europeo, in Europa e dir. priv., 2005, pp. 977-1012; R. Pardolesi e Ponzanelli (a cura di), "I 25 anni di products liability", in Danno e resp., 2012.

6 Sul punto v. R. Pardolesi e Caruso, Per una storia della Direttiva 1985/374/CEE, in Danno e resp., 2012; si veda anche The Blu Guide on the Implementation of EU Product Rules, pubblicata dalla Comm. nel 2014, p. 6. Per una esaustiva illustrazione riferita al contesto statunitense v. Owen, Montgomery and Davis, Product Liability and Safety, IV ed., New York, 2010, p. 2.

${ }^{7}$ Con particolare riferimento alla responsabilità del produttore Tassone, Responsabilità da prodotto e nesso di causa, "I 25 anni di products liability", in Danno e resp., 2012, p. 21. Più in generale, sul problema della causalità, Franzoni, L'illecito, I, II ed., Milano, 2010, p. 61; Pucella, La causalità incerta, Torino, 2007; Nocco, Il "sincretismo causale” e la politica del diritto: spunti dalla responsabilità sanitaria, Torino, 2010; Capecchi, Il nesso di causalità. Dalla condicio sine qua non alla responsabilità proporzionale, Padova,2012. 
del Restatement Second del 1965 il Chapter I del Restatement (Third) of Torts del 1998, nei quali sono individuate le linee guida ed i principi generali che emergono del diritto statunitense.

Nei sistemi di norme concepiti al fine di regolare il problema dell'allocazione dei costi derivanti dai danni provocati da prodotti di larga diffusione si compendiano due esigenze distinte, ma strettamente correlate: quella di offrire una adeguata tutela agli utilizzatori e quella di promuovere un'efficiente funzionamento del mercato ed una equilibrata competizione tra gli imprenditori che operano in uno spazio economico comune ${ }^{8}$. Sotto il primo profilo la maggiore rispondenza della disciplina alle esigenze di tutela del danneggiato emerge laddove ad esso non è richiesto di provare una colpa in capo al danneggiante, ma solamente il carattere difettoso del prodotto, ossia la circostanza che questo non possieda la "sicurezza che ci si può legittimamente attendere tenuto conto di tutte le circostanze" (art. 6, dir. 85/374/CEE) o, secondo una formula propria del diritto statunitense, che il prodotto risulti unreasonably dangerous (section $402 \mathrm{~A}, 1$ del Restatement Second). In una simile prospettiva al danneggiato è sufficiente provare il difetto, il danno e l'esistenza di un rapporto causale tra di essi, mentre grava sul produttore l'onere di provare $\mathrm{i}$ fatti che possono escludere la sua responsabilità per $\mathrm{i}$ danni causalmente riconducibili all'utilizzo del prodotto. Il fabbricante, quindi, è chiamato a dimostrare la non difettosità del prodotto, fornendo una prova che nel sistema statunitense si risolve nella dimostrazione della conformità del prodotto stesso alle regole comunemente riconosciute ed adottate dai fabbricanti (state of the art defense) ${ }^{9}$ e, in quello ideato dalla dir. 85/374/CEE impone di provare la sussistenza di quelle cause di esclusione della responsabilità tassativamente indicate dall'art. 6 , dir. 85/374/CEE.

È evidente che in un sistema nel quale la responsabilità del fabbricante presuppone l'accertamento della difettosità del prodotto e quindi della sua non rispondenza ai requisiti di sicurezza che dovrebbero essere presenti in un modello ideale di prodotto ragionevolmente sicuro si impone l'esigenza di individuare criteri oggettivi e condivisi che consentano di circostanziare nella misura più stringente possibile il generico riferimento alla "sicurezza che ci si può legittimamente attendere tenuto conto di tutte le circostanze" a cui si riferisce il legislatore dell'Unione Europea o alla irragionevole pericolosità (unreasonably dangerous) che costituisce il parametro di riferimento assunto dal "diritto vivente" nordamericano. In altri termini, quelle esigenze di armonizzazione giuridica che costituiscono il presupposto indispensabile per un efficiente funzionamento del mercato e un equilibrato svolgimento della competizione tra gli operatori economici postulano la creazione e la diffusione di "regole tecniche" nelle quali il legislatore trasfonda il concetto di stato dell'arte delineando con dettaglio e precisione quel modello ideale di prodotto che costituisce il parametro per valutare in concreto la difettosità dei singoli prodotti dal cui utilizzo derivi un danno. Ciò dovrebbe comportare una correlativa riduzione del potere discrezionale del giudice, il quale, in linea di principio, dovrebbe essere chiamato ad allinearsi alle valutazioni espresse dal legislatore e compiutamente definite negli standards tecnici.

\footnotetext{
${ }^{8} \mathrm{Nel}$ primo Considerando della dir. 85/374/CEE si osserva che "il riavvicinamento delle legislazioni nazionali in materia di responsabilità del produttore per i danni causati dal carattere difettoso dei prodotti è necessario perché le disparità esistenti fra tali legislazioni possono falsare il gioco della concorrenza e pregiudicare la libera circolazione delle merci all'interno del Mercato comune determinando disparità nel grado di protezione del consumatore contro i danni causati alla salute e ai suoi beni da un prodotto difettoso"'. Sul punto v. Alpa, L'attuazione della direttiva nei paesi della CEE, in La responsabilità del produttore a cura di Alpa, Bin, Cendon, XIII, in Tratt. dir. comm.edir. pubbl. econ. diretto da Galgano, cit.,p. 17 ss.

9 Nella Illustration 2 del Restatement Third, Torts: Products Liability section 2, p. 20, si mette in luce che le molteplici definizioni del c.d. stato dell'arte (la tecnologia più avanzata, il livello più evoluto di conoscenze scientifiche, lo standard di qualità media adottato dalla produzione industriale) portano a concludere che si tratti di un concetto vago, che si presta ad interpretazioni discrezionali e caratterizzate da un elevato grado di approssimazione.
} 
Il danno da "prodotto conforme": le soluzioni europee e statunitensi nella prospettiva del Transatlantic Trade and Investment Partnership (T.T.I.P.)

Nel contesto normativo attuale, in effetti, risulta sempre più diffusa una "normazione tecnica" che definisce compiutamente le caratteristiche strutturali e gli standards di sicurezza dei prodotti e rappresenta un punto di riferimento imprescindibile al fine di valutarne la sicurezza. Proprio le regole che governano la sicurezza dei prodotti, pertanto, risultano determinanti al fine di promuovere quegli obiettivi di efficiente funzionamento del mercato ed equilibrata competizione tra gli imprenditori che operano in uno spazio economico comune; obiettivi che, tanto il legislatore europeo quanto quello statunitense, dichiarano esplicitamente di perseguire ${ }^{10}$.

\section{GLI STANDARDS ARMONIZZATI DI SICUREZZA DEI PRODOTTI NELL'UNIONE EUROPEA E NEGLI STATI UNITI}

Sia nella legislazione dell'Unione Europea, sia in quella statunitense, occorre operare una netta distinzione tra prodotti che sono stati interessati dal fenomeno della normazione tecnica e quelli che, invece, ne sono rimasti estranei. In quest'ultima categoria ricadono, ad esempio, i vestiti ${ }^{11}$, le scarpe ${ }^{12}$, larga parte dei complementi d'arredamento ${ }^{13}$, alcuni oggetti di cancelleria. Per questi prodotti il legislatore non detta norme che impongano l'adozione di particolari caratteristiche tecniche e costruttive; il fatto che il prodotto offra un livello di sicurezza ragionevole, quindi, può essere ricavato facendo riferimento alle ragionevoli aspettative del consumatore ed alle regole dell'arte.

Per altre categorie di beni, invece, la regola generale secondo cui deve considerarsi difettoso il prodotto che non garantisca la sicurezza che il consumatore può "legittimamente attendere" è stata rigorosamente circostanziata: infatti nell'Unione Europea e, ancor prima, negli Stati Uniti, il legislatore ha predisposto complessi sistemi di regole che definiscono dettagliatamente le caratteristiche che determinati prodotti debbono possedere per poter essere considerati sicuri. A questo proposito gli interpreti statunitensi hanno utilizzato l'espressione particolarmente incisiva di "intrusione" di regole tecniche nelle norme giuridiche proprio per evidenziare come, con riferimento a determinate categorie di prodotti, le regole dell'arte siano state trasfuse direttamente in norme di legge che contengono dati tecnici o che rinviano a standards predisposti da enti di normalizzazione accreditati. Queste previsioni costituiscono un fondamentale elemento unificante che consente di realizzare l'armonizzazione della responsabilità del produttore in un sistema giuridico unitario composto da differenti ordinamenti statali; esse, infatti contengono "pre-valutazioni" tecniche che incidono profondamente sull'applicazione delle norme in tema di responsabilità civile ${ }^{14}$

${ }^{10} \mathrm{Nel}$ primo considerando della direttiva si osserva che "il riavvicinamento delle legislazioni nazionali in materia di responsabilità del produttore per i danni causati dal carattere difettoso dei prodotti è necessario perché le disparità esistenti fra tali legislazioni possono falsare il gioco della concorrenza e pregiudicare la libera circolazione delle merci all' interno del Mercato comune determinando disparità nel grado di protezione del consumatore contro i danni causati alla salute e ai suoi beni da un prodotto difettoso". Sul punto v. Alpa, L'attuazione della direttivaneipaesidella CEE, cit.,p. 17 ss.

${ }^{11}$ Trib. Roma 12 maggio 2004, in Resp. civ. eprev., 2005, p. 217, con nota di Della Bella,Laresponsabilità del produttore ditutesportive.

${ }^{12}$ Tra le decisioni che hanno sancito la responsabilità del produttore di indumenti e, opportunamente, non hanno fatto riferimento a particolari discipline tecniche in materia di sicurezza si vedano Trib. Roma 12 maggio 2004, cit., relativa ai danni provocati da una tuta realizzata con materiali infiammabili; Trib. La Spezia 27 ottobre 2005, in Foro it., 2005, I, c. 3500; in Corr. merito, 2006, 2, p. 177, con nota di Cabella Pisu, Il costo di una caduta ... dagli stivali, in materia di danni provocati da una calzatura che presentava un difetto di fabbricazione tale da cagionare la caduta della persona che la indossava.

${ }^{13}$ Sul punto v. Trib. Milano 13 aprile 1995, in Danno e resp., 1996, p. 381, con nota di Ponzanelli, Crollo di un letto a castello: responsabilità del produttore-progettista e del montatore.

${ }^{14}$ Come si osserverà diffusamente, le caratteristiche dei prodotti e le modalità di svolgimento delle attività sono sempre più di frequente regolate da disposizioni che incorporano valutazioni tecniche assai complesse, oppure 
in quanto forniscono uno standard unico sulla base del quale valutare la difettosità del prodotto delle diverse giurisdizioni statali.

Nell'Unione Europea l'obbligo di immettere sul mercato prodotti sicuri viene sancito in termini generali, per tutte le tipologie di prodotto, dalla dir. 2001/95/CE (artt. 1,2 e 3) (c.d. legislazione orizzontale) ed è costantemente ribadito nella c.d. legislazione verticale ${ }^{15}$, ossia nelle singole direttive specificamente riferite a determinate categorie di beni, come, ad esempio, i farmaci (dir. 2001/83/CE) ${ }^{16}$, i presidi medico-chirurgici (dir. 2007/47/CE) ${ }^{17}$, i cosmetici (dir. 76/768/CEE) ${ }^{18}$, il materiale elettrico (dir. 2006/95/CE) ${ }^{19}$, le bombole a gas, gli autoveicoli (dir. 2007/46/CE), i motoveicoli a due e tre ruote (dir. 2002/24/CE), gli pneumatici (dir. 1992/ 23/CE; dir. 2001/43/CE; 2005/11/CE) ed i giocattoli (dir. 2009/48/CE) ${ }^{20}$. L'obbligo di sicurezza enunciato in termini generali dalla legislazione "verticale" e dalla legislazione "orizzontale" (dir. 2001/95/CE) è assai di frequente circostanziato da "norme tecniche armonizzate". Proprio mediante questo strumento si realizza la c.d. armonizzazione giuridica che il legislatore dell'Unione Europea ha inaugurato a partire dalla c.d. "direttiva bassa tensione" (dir. 73/ 23/CEE del 19 febbraio 1973) ${ }^{21}$ e ulteriormente sviluppato con il

rinviano a vere e proprie "regole tecniche" predisposte da enti accreditati e costantemente aggiornate in armonia con il progresso scientifico e tecnologico. Solo per fare alcuni esempi, basti pensare all' elenco delle "sostanze ammesse" per la produzione dei cosmetici, ai requisiti di omologazione richiesti per la immissione in commercio degli autoveicoli, alle regole che fissano soglie di esposizione alle onde elettromagnetiche emesse dai telefoni cellulari, alle norme tecniche imposte per la sicurezza di prodotti come le biciclette, le scale, gli accendini, le bombole a gas (Carnevali, Prevenzione e risarcimento nelle direttive comunitarie sulla sicurezza dei prodotti, cit., p. 11). Anche le norme pubblicistiche sulla produzione e la manipolazione degli alimenti risultano talvolta estremamente dettagliate e indicano con precisione a quali standards qualitativi sia necessario adeguarsi. Solo per fare alcuni esempi, per quanto concerne gli integratori alimentari il principio della "lista positiva" impone l'obbligo di utilizzare per la fabbricazione di integratori alimentari solo vitamine e minerali elencati negli allegati al d. lgs. n. 169/2004); con riferimento al latte il d.p.r. 54/1997 enuncia dettagliati requisiti che disciplinano le procedure di pastorizzazione e fissano le qualità organolettiche del prodotto (sul punto cfr. Al Mureden, I danni dal consumo di alimenti tra legislazione di settore, principio diprecauzione responsabilità civile, in questarivista,2011,p. 1495 ss.). A queste regole occorre aggiungere, da ultimo, quelle relative all'etichettatura dei prodotti alimentari contenute nel reg. Reg. (UE) 1169/2011, applicabile a decorrere dal 13 dicembre 2014.

${ }^{15}$ Le regole sulla sicurezza generale dei prodotti costituiscono una normativa "orizzontale" che si affianca a quelle più specifiche (c.d. norme verticali). I rapporti tra legislazione orizzontale e legislazione verticale sono regolati dai criteri della sussidiarietà e complementarietà. Pertanto la disciplina generale sulla sicurezza dei prodotti non si applica ove sia presente una disciplina specifica (sul punto v. Carnevali, Prevenzione e risarcimento nelle direttive comunitarie sulla sicurezza dei prodotti, cit., p. 11; Cavallo, sub art. 102, in Codice del consumo e norme collegate a cura di Cuffaro, III ed., Milano, 2012, p. 575; Cordiano, Sicurezza dei prodottietutelapreventivadeiconsumatori, Padova,2005,p.43ss.).

${ }^{16}$ La Direttiva è stata attuata in Italia dal d. lgs. n. 219/2006.

${ }^{17}$ Per un'analitica illustrazione delle discipline di settore previste in tema di presidi medico chirurgici (come ad esempio le protesi mammarie, le lenti a contatto, ecc.) Querci, Protesi mediche tra regolamentazione di sicurezza e responsabilità da prodotto: l'onere della prova tutela il consumatore, in Danno e resp., 2008, p. 290; Di Loreto, I dispositivi medici tra regolamentazione di sicurezza e responsabilità, in Danno e resp., 2007, p. 193.

${ }^{18}$ La Direttiva è stata attuata in Italia dalla 1. n. 713/1986. L'intera disciplina dei cosmetici è stata completamente rinnovata dal Reg. (Ce) n. 1223/2009, in vigore dal 11 luglio 2013.

${ }^{19}$ La direttiva ha sostituito la precedente dir. 73/23/CEE; 1. n. 791/1977.

${ }^{20}$ In precedenza la materia era regolata dalla dir. 88/378/CEE attuata mediante d. lgs.n.313/1991.

${ }^{21}$ In questa direttiva l'uniformazione delle normative tecniche nazionali fu attuata mediante una sinergia tra la legge - che disciplinava direttamente soltanto gli obiettivi di sicurezza da raggiungere-e le cosiddette specifiche tecniche mediante le quali gli obiettivi indicati dal legislatore venivano messi a fuoco attraverso il rinvio ad una normazione tecnica di natura consensuale, la cui elaborazione era affidata al CENELEC, un'associazione di natura privatistica costituita nel 1973, che emanava standards validi a livello europeo. La decisione di aderire alle specifiche tecniche del CENELEC e fabbricare prodotti ad esse conformi assicurava una presunzione di conformità agli obiettivi di sicurezza sanciti dalla dir. 73/23/CEE e consentiva ai costruttori di risultare esonerati dall'onere della prova circa la sicurezza del prodotto Cagli, Organizzazione procedure dell'attività amministrativa tecnica nel settore dei prodotti industriali, in Aa.Vv., La normativa tecnica industriale: amministrazione e privati nella normativa tecnica e nella certificazione dei prodotti industriali a cura di Andreini, Bologna, 1995, p. 171, osserva che, in assenza di norme armonizzate elaborate dal CENELEC, era consentito adottare modalità 
Il danno da "prodotto conforme": le soluzioni europee e statunitensi nella prospettiva del Transatlantic Trade and Investment Partnership (T.T.I.P.)

c.d. "nuovo approccio"22 (dir. 83/189/CEE), dando vita ad un articolato sistema di direttive settoriali riguardanti determinate tipologie di prodotti e particolari fattispecie di rischio. Così, per ogni categoria di prodotti regolati da una direttiva, la Commissione europea conferisce un mandato agli Enti europei di Normazione (ossia al CEN (Comitato europeo di normalizzazione), al CENELEC (Comitato europeo di normalizzazione elettrotecnica) ${ }^{23}, \mathrm{o}$ all'ETSI (Istituto europeo norme e telecomunicazioni) ${ }^{24}$ che operano di concerto con il Comitato permanente (artt. 5 e 6, dir. 34/98/CE). Le norme tecniche elaborate ed approvate dagli organismi europei di normalizzazione vengono pubblicate, in seguito, sulla Gazzetta Ufficiale della Comunità Europea (G.U.C.E.) e nella Gazzetta Ufficiale di ogni Paese aderente $^{25}$.

Da questo momento la loro adozione come norme tecniche nazionali è obbligatoria per gli enti di normalizzazione istituiti in ciascuno degli Stati membri che dovranno riprodurre a livello nazionale le norme tecniche corrispondenti a quelle emanate dagli enti di normazione europei ${ }^{26}$. Così, ad esempio, nel nostro ordinamento gli enti di normalizzazione

produttive che corrispondevano alla buona tecnica secondo i criteri elaborati in campo internazionale. Con la direttiva "bassa tensione", invece, si è dato vita per la prima volta ad un approccio mediante il quale la Comunità Europea ha imposto ai produttori solamente l'obiettivo di immettere sul mercato prodotti conformi "alla regola dell'arte CEE", offrendo, nel contempo, un ventaglio di possibilità per consentire agli stessi produttori di provare la conformità dei loro prodotti a tale regola.

${ }^{22}$ Osserva Carnevali, Prevenzione e risarcimento nelle direttive comunitarie sulla sicurezza dei prodotti, cit., pp. 5-6, che l'utilizzo delle norme tecniche da parte della Comunità ha avuto inizio con la Risoluzione del Consiglio del 7 maggio 1985. Il c.d. nuovo approccio è retto da quattro principi fondamentali sanciti dal Consiglio: l'armonizzazione legislativa deve limitarsi ai requisiti fondamentali in materia di sicurezza (o ad altri requisiti di interesse collettivo) che i prodotti commercializzati devono soddisfare per essere messi in libera circolazione nella Comunità; il compito di elaborare specifiche tecniche armonizzate deve essere affidato a organismi competenti per la normalizzazione industriale e accreditati dalla dir. 83/189/CE; le specifiche tecniche non debbono avere carattere obbligatorio e rivestono carattere volontario; le amministrazioni sono tenute a riconoscere ai prodotti fabbricati conformemente alle norme armonizzate una presunzione di conformità ai requisiti fondamentali stabiliti dalla direttiva. Qualora il produttore non fabbrichi attenendosi a tali norme, incomberà su di lui un dovere di dimostrare la conformità di tali prodotti ai requisiti fondamentali (sul punto si vedano le indicazioni riportate all'indirizzo http://europa.eu/legislation_summaries/internal_market/single_market_for_goods/technical_harmonisation/l21001a_it.htm). Si vedano, inoltre, le osservazioni di Salmoni, Le norme tecniche, Milano, 2001, p. 326, la quale precisa che le direttive prevedono come regola un'armonizzazione totale: quindi solamente i prodotti conformi possono essere commercializzati. In definitiva le direttive fissano i requisiti essenziali di sicurezza e le norme armonizzate impongono le specifiche tecniche secondo un modello definito di "rinvio agli standards".

${ }^{23}$ Il CENELEC (http://www.cenelec.eu) è il Comitato europeo per la normalizzazione elettrotecnica; a questo ente è affidato il compito di predisporre gli standards tecnici mediante i quali viene definita la sicurezza ragionevole dei prodotti elettrotecnici.

${ }^{24} \mathrm{Il} \mathrm{CEN} \mathrm{(http://www.cen.eu/cen/AboutUs/Pages/default.aspx)} \mathrm{è} \mathrm{l'organismo} \mathrm{che,} \mathrm{in} \mathrm{virtù} \mathrm{dei} \mathrm{poteri} \mathrm{conferiti} \mathrm{dalla} \mathrm{dir.}$ 98/34/CE, emana gli standards tecnici a livello europeo con riferimento a tutte le attività produttive eccetto quella della tecnologia elettronica la cui competenza è riservata al CENELEC ( $h t t p: / / w w w . c e n e l e c . e u$ ) e quella delle telecomunicazioni, di competenza dell'ETSI (http://www.etsi.org).

${ }^{25}$ In Europa il legislatore ha ritenuto che le norme tecniche potessero costituire uno strumento di grande utilità economica e sociale. Tali norme, elaborate su richiesta della Commissione Europea e citate in appositi elenchi nella G.U.C.E., vengono dette "armonizzate". Al riguardo si vedano le indicazioni riportate nel sito ufficiale dell'U.N.I. (http://www.uni.com/) e le osservazioni di Salmoni, Le norme tecniche, cit., p. 326; Cagli, Organizzazione procedure, cit., p. 175; Bellisario, sub artt. 101-113, in Codice del consumatore-Comm. a cura di Alpa, Rossi Carleo, Napoli, 2005, pp. 696-697.

${ }^{26}$ In un primo momento le norme tecniche armonizzate si svilupparono per assecondare la necessità - connaturata allo sviluppo della produzione industriale in serie - di circostanziare il vago riferimento allo stato dell'arte dando vita a regole tecniche standardizzate ed uniformi. Tale esigenza si è manifestata nei diversi ordinamenti europei a partire dai primi anni del Novecento. Anche solo in una prospettiva nazionale, l'esigenza di produrre beni ed erogare servizi secondo standards qualitativi uniformi fu assecondata, inizialmente, attraverso la creazione di enti deputati alla produzione di norme tecniche uniformi e armonizzate. Il Comitato Elettrotecnico Italiano (C.E.I.), fondato nel 1909, ha elaborato norme che definivano la buona tecnica per i prodotti, per i processi e per gli impianti produttivi, costituendo il riferimento per la presunzione di conformità alla "regola dell'arte". In seguito per svolgere attività normativa tecnica in tutti i settori esclusi quelli elettromagnetico ed elettronico (che ricadono 
nazionali quali 1'U.N.I. (Ente Nazionale Italiano di Unificazione) ${ }^{27}$ ed il C.E.I. (Comitato Elettrotecnico Italiano) sono tenuti a riprodurre le regole tecniche armonizzate elaborate dagli enti di normalizzazione europei; lo stesso accade negli altri ordinamenti che aderiscono all'Unione Europea e nei quali gli enti di normalizzazione nazionali riproducono il contenuto delle norme tecniche elaborate dal CEN o dal CENELEC ${ }^{28}$.

In questo modo la sicurezza dei prodotti viene definita secondo uno standard comune a tutti i paesi che fanno parte dell'Unione Europea.

La situazione appena descritta con riferimento all'Unione Europea si presenta in termini analoghi nel contesto statunitense. Anche in quell'ordinamento, infatti, l'obiettivo di garantire ai consumatori una protezione contro i rischi irragionevoli associati all'utilizzo dei prodotti è stato perseguito in termini generali attraverso l'introduzione del Consumer Product Safety Act (1972); al tempo stesso, con riferimento a categorie di prodotti particolari, il legislatore federale è intervenuto con discipline specifiche: è il caso, ad esempio, del Federal Food, Drug, and Cosmetic Act (1906), con il quale è stata regolamentata la sicurezza dei farmaci, degli alimenti e di altre categorie di prodotti dai quali possono scaturire pericoli per la salute $^{29}$, dell'Highway Safety Act (1966), che è intervenuto a regolare la sicurezza della circolazione stradale e le caratteristiche costruttive degli autoveicoli, del Communications Act

nella competenza del C.E.I.) è stato istituito l'U.N.I. (Ente Nazionale Italiano di Unificazione), un'associazione privata senza fine di lucro fondata nel 1921 e riconosciuta dallo Stato e dall'Unione Europea, che studia, elabora, approva e pubblica le norme tecniche volontarie - le cosiddette "norme U.N.I." - in tutti i settori industriali, commerciali e del terziario (tranne in quelli elettrico ed elettrotecnico). Il percorso brevemente descritto si è sviluppato parallelamente in molti altri Stati. Così, nei diversi Paesi europei, anche successivamente all'istituzione della Comunità Economica Europea operavano enti di normazione che predisponevano regole non necessariamente armonizzate con quelle di altri Stati. Per un lungo periodo, quindi, le norme tecniche emanate dagli enti di normazione sono state concepite principalmente in un'ottica nazionale ed hanno rivestito un'importanza decisamente marginale sotto il profilo dei riflessi sui rapporti privatistici. In altre parole ogni ordinamento disponeva di enti di normalizzazione che in modo autonomo specificavano il concetto di stato dell'arte (Salmoni, Le normetecniche, cit.,p. 228).

${ }^{27} \mathrm{Nel}$ sito ufficiale dell'U.N.I-Ente Nazionale Italiano di Unificazione (http://www. uni.com/it) si legge che esso "è un'associazione privata senza scopo di lucro" che "svolge attività normativa in tutti i settori industriali, commerciali e del terziario ad esclusione di quello elettrico ed elettrotecnico di competenza del C.E.I.-Comitato Elettrotecnico Italiano". "Il ruolo dell'U.N.I. quale Organismo nazionale italiano di normazione", continua la pagina di presentazione, "è stato riconosciuto dalla dir. 83/189/CEE del marzo 1983, recepita dal Governo Italiano con la 1. n. 317 del 21 giugno 1986”. L’U.N.I. partecipa, in rappresentanza dell'Italia, all'attività normativa degli organismi sovranazionali di normazione: I.S.O. (InternationalOrganizationforStandardization)eC.E.N.(ComitéEuropéendeNormalisation).Lenorme armonizzate europee assumono un ruolo di preminenza e confinano l'operatività delle regole nazionali ad una funzione residuale e sussidiaria che viene in considerazione solo laddove la regola europea sia assente. Pertanto gli enti di normalizzazione nazionali (U.N.I. e C.E.I) - il cui riconoscimento ufficiale nei settori di rispettiva competenza era già stato sancito (1. n. 317/1986, attuativa della dir. 83/189/CEE) - conservano il loro ruolo, ma assumono una posizione subordinata rispetto a quelli europei:essi, infatti, sono tenuti a trasporre nel contesto nazionale gli standards tecnici elaborati dagli enti di normalizzazione europei. Ciò non esclude l'esistenza di ambiti nei quali gli enti di normalizzazione nazionali possono predisporre regole tecniche con una valenza limitata al contesto interno; tali regole, inogni caso, dovranno essere verificate sulla base diuna procedura a livello comunitario, gestita dalla Commissione e assistita da un comitato permanente di responsabili delle amministrazioni nazionali.

${ }^{28}$ Sul problema della certificazione di conformità Bugiolacchi, Sicurezza dei prodotti e certificazione: la responsabilità contrattuale degli organismi di valutazione della conformità - [Product safety and certification: the contractual liability of the conformity assessment parties], in Resp. civ. prev., 2013, p. 610 ss.

${ }^{29}$ L'articolata disciplina in materia di sicurezza alimentare è stata ulteriormente incrementata a seguito della approvazione nel 2011 del Food Safety Modernization Act (F.S.M.A.), con il quale sono state implementate le misure di sicurezza funzionali a garantire che il cibo importato dall'estero negli Stati Uniti presenti le caratteristiche di qualità richieste dalla legislazione federale e dai regolamenti emanati dalla F.D.A. (cfr. amplius in http://www.fda.gov/ downloads/Food/FoodSafety/FSMA/UCM277713.pdf). In argomento v. Magli, La sicurezza alimentare tra norme preventive, obblighi risarcitori ed autoresponsabilità del consumatore. Sistema italiano e modello statunitense a confronto, Bologna, 2013. 
Il danno da "prodotto conforme": le soluzioni europee e statunitensi nella prospettiva del Transatlantic Trade and Investment Partnership (T.T.I.P.)

del 1934, nel quale sono contenute importanti disposizioni connesse alla protezione della salute ed al pericolo dell'esposizione a campi elettromagnetici.

Per ciascuna delle legislazioni indicate è stata istituita una particolare Agency legittimata ad emanare regulations che specificano i generici obblighi di sicurezza sanciti nelle fonti legislative "di primo livello", definendo le caratteristiche tecniche necessarie per conseguire un grado di sicurezza ragionevole. Così la Consumer Product Safety Commission ${ }^{30}$ definisce gli standards di sicurezza di diversi prodotti soggetti alla disciplina generale sulla sicurezza prevista dal Consumer Product Safety Act; la Food and Drug Administration (F.D.A. $)^{31}$ emana gli standards tecnici che circostanziano le previsioni contenute nel Federal Food, Drug, and Cosmetic Act; la National Highway Traffic Safety Agency (N.H.T.S.A.) ${ }^{32}$ definisce i requisiti di sicurezza degli autoveicoli specificando i principi sanciti nell' Highway Safety Act. Ciascuna delle Agencies indicate emana a cadenza annuale regulations che definiscono le caratteristiche di sicurezza relative alle differenti tipologie di prodotti che rientrano nella loro competenza. Tali regulations vengono pubblicati sul Federal Register, che assolve una funzione assimilabile a quella della Gazzetta ufficiale ${ }^{33}$; essi, inoltre, sono compendiati in un testo unico federale: il Code of Federal Regulations (C.F.R.) ${ }^{34}$, che presenta una struttura corrispondente a quella dello United States Code (U.S.C.). Anch'esso, pertanto, è suddiviso in 51 titles, che corrispondono alle medesime aree tematiche secondo le quali è organizzato lo United States Code (U.S.C.) e sono a loro volta suddivisi in subtitles e chapters $^{35}$.

Proprio la previsione di standards di sicurezza armonizzati a livello federale costituisce l'elemento di aggregazione ed armonizzazione intorno al quale in larga misura si ricompongono le diversità di disciplina che caratterizzano la legislazione dei singoli Stati. Come osservato, il legislatore federale, infatti, fissa requisiti generali di sicurezza con riferimento alle diverse categorie di prodotti ed istituisce Agenzie federali (Agencies) legittimate ad emanare periodicamente complesse normative tecniche che specificano nel dettaglio le caratteristiche necessarie affinché i prodotti possono essere considerati sicuri. Lo stesso legislatore federale, poi, attraverso le cosiddette clausole di preemption $^{36}$ sancisce la prevalenza degli

\footnotetext{
${ }^{30}$ Le funzioni della Consumer Product Safety Commission sono incisivamente illustrate nel sito ufficiale (http://www.cpsc.gov/about/about.html) in cui viene chiarito che "The U.S. Consumer Product Safety Commission is charged with protecting the public from unreasonable risks of injury or death from thousands of types of consumer products under the agency's jurisdiction. The CPSC is committed to protecting consumers and families from products that pose a fire, electrical, chemical, or mechanical hazard or can injure children. The CPSC's work to ensure the safety of consumer products - such as toys, cribs, power tools, cigarette lighters, and household chemicals - contributed significantly to the 30 percent decline in the rate of deaths and injuries associated with consumer products over the past 30 years".

${ }^{31}$ Per un'analitica illustrazione delle origini, della struttura e delle funzioni dell'Agency si rinvia al sito ufficiale http://www.fda.gov/default.htm.

${ }^{32}$ Origini, struttura e funzioni dell'Agency sono diffusamente illustrate nel sito ufficiale http://www.nhtsa.gov/.

${ }^{33} \mathrm{Cfr}$. il sitoufficiale $h t t p: / / w w w$.archives.gov/federal-register/the-federal-register/.

${ }^{34} \mathrm{Cfr}$. il sito ufficiale $h t t p: / / w w w$.archives.gov/federal-register/cfr/.

${ }^{35} \mathrm{Cfr}$. il sito ufficiale http://www.gpo.gov/fdsys/browse/collectionCfr.action? collection Code $=$ CFR.

${ }^{36}$ Con questa espressione si indica la prevalenza delle leggi costituzionali e federali su quelle statali. Nello specifico contesto della responsabilità del produttore la preemption doctrine è stata applicata al fine di sancire la prevalenza degli standards federali su quelli previsti dalle singole giurisdizioni e costituisce uno strumento fondamentale per perseguire l'obiettivo della armonizzazione degli standards di sicurezza nei diversi stati. In argomento v. McGarity, The Preemption War: When Federal Bureaucracies Trump Local Juries, New Haven London, 2008; Untereiner, The preemption defense in tort actions: Law Strategy and Practice Washington D.C., 2008; O'Reilly, Federal Preemption of State and Local Law, Chicago, 2006; Dinh, Rassessing the law of Preemption, 88 Geo L.J. 2085 (2000); Goldsmith, Statury Foreign Affairs Preemption, Sup. Ct. Rev. 175 (2000); Sharkey, Products Liability Preemption: an Institutional Approch, 76 Geo. Wash. Law Rev., 449 (2008); Davis, On Restating Products Liability Preemption, 74 Brooklyn Law Rev. 759 (2009). Per un'esaustiva illustrazione in lingua italiana Querci, Responsabilità da prodotto negli Usa e in Europa. Le ragioni di un revirement "globale”, in Nuova giur. civ. comm., 2011, p. 118.
} 
standards federali su quelli statali, chiarisce in modo esplicito (express preemption) o implicito (implied preemption) se lo standard federale assume la valenza di limite massimo di sicurezza (c.d. ceiling), rispettato il quale non è possibile configurare un'azione di responsabilità, ovvero se esso costituisce solo un limite minimo (c.d. floor), il cui rispetto non esime il produttore dalla responsabilità per i danni riconducibili all'utilizzo del prodotto.

In un sistema così congegnato, il giudice chiamato a decidere riguardo alla responsabilità del produttore è tenuto ad "allinearsi" alle indicazioni fornite dal legislatore federale con riferimento alla sicurezza dei prodotti e, in definitiva, ad emettere una decisione che risulti coerente con le scelte operate da quest'ultimo. Proprio il rigoroso rispetto del "percorso predefinito" tracciato dalla legislazione federale sulla sicurezza dei prodotti costituisce il presupposto per addivenire ad un elevato grado di armonizzazione, pur in presenza di una evidente frammentazione delle regole che governano la responsabilità del produttore, di quelle in materia di class action e di quelle che regolano i c.d. punitive damages nei diversi ordinamenti statali.

Anche il sistema delineato dal legislatore nell’Unione Europea si caratterizza per la presenza di un "anello di congiunzione" 37 tra la disciplina della sicurezza dei prodotti e quella della responsabilità del produttore che può essere colta con evidenza proprio con riferimento a quelle categorie di beni interessate dalla normazione tecnica standardizzata.

\section{IL PROBLEMA DEL DANNO DA PRODOTTO CONFORME E LA DISTINZIONE TRA PRODOTTO DANNOSO E PRODOTTO DIFETTOSO}

Il problema dell'allocazione dei costi derivanti dai danni provocati dall'utilizzo di prodotti fabbricati in serie presenta una specificità assai più ampia rispetto a quello del risarcimento dei danni provocati da prodotti difettosi al quale sia la disciplina legislativa, sia l'interprete hanno riservato un'attenzione preminente e, sotto alcuni profili, assorbente. Il carattere insicuro, e quindi difettoso, del prodotto che cagiona un danno è assunto quale presupposto indefettibile della responsabilità del fabbricante; sembra, pertanto, che tale responsabilità possa scaturire solo laddove una "anomalia" che si manifesta nell'ambito della progettazione, della fabbricazione o del corredo di informazioni che dovrebbero accompagnare il prodotto determini una irragionevole insicurezza dalla quale scaturisce un danno. Cionondimeno, è emersa da tempo una piena consapevolezza circa la sussistenza dei rilevanti pericoli che possono derivare dall'utilizzo di prodotti pienamente conformi agli standards di sicurezza individuati dal legislatore al fine della loro immissione in commercio. Così, il consumo di prodotti derivati dal tabacco, l'utilizzo di autoveicoli, l'esposizione alle onde elettromagnetiche emesse dal telefono cellulare, l'ingestione di alimenti la cui conservazione ed igiene è conseguita anche attraverso la presenza al loro interno di sostanze chimiche potenzialmente dannose per la salute, infine l'assunzione di farmaci che, seppur indispensabili, contengono sostanze capaci di scatenare rilevanti effetti collaterali, costituiscono solo alcuni esempi di prodotti che conservano un ampio margine di dannosità, pur risultando pienamente conformi alle prescrizioni legali che ne regolano la sicurezza e, in un certo senso, possono essere considerati dannosi proprio perché perfettamente conformi e funzionali alle finalità per le quali vengono utilizzati.

${ }^{37}$ L'espressione è di Carnevali, Prevenzione e risarcimento nelle direttive comunitarie sulla sicurezza dei prodotti, cit., p. 12. 
Il danno da "prodotto conforme": le soluzioni europee e statunitensi nella prospettiva del Transatlantic Trade and Investment Partnership (T.T.I.P.)

Sotto questo profilo emerge la fondamentale importanza assunta dalla distinzione tra prodotto difettoso e prodotto dannoso. Nella prima categoria ricadono i prodotti che risultino difformi rispetto alle caratteristiche delineate dalle norme tecniche standardizzate o, ove queste non siano presenti, definite dallo stato dell'arte; nella seconda, invece, rientrano i prodotti dai quali possano scaturire rilevanti danni per coloro che li utilizzano o vengono a contatto con essi. Le due categorie coincidono solo occasionalmente: il prodotto non conforme rispetto alle caratteristiche tecniche prescritte dal legislatore, e quindi difettoso, può sicuramente assumere in alcune circostanze un carattere dannoso (si pensi, ad esempio, all' automobile il cui difetto provochi un incidente o al telefono cellulare difettoso che, peripotesi, esploda durante il funzionamento), ma potrebbe, in altre, risultare — quasi paradossalmente - completamente privo di rischi (si pensi, ad esempio, all'automobile o al telefono cellulare non funzionante e per questo motivo assolutamente privi di rischi); al tempo stesso il prodotto conforme alle caratteristiche tecniche prescritte dalla legislazione sulla sicurezza potrebbe conservaresignificativi margini di dannosità proprio perché perfettamente funzionante. In quest'ultimo caso l'utilizzatore o colui che entra in contatto con il prodotto si troverebbe a subire un danno derivante da un prodotto pienamente conforme agli standards legislativi di sicurezza ed utilizzato secondo modalità appropriate, ma, cionondimeno, caratterizzato da una elevata capacità di produrre danni.

Nel nostro ordinamento giuridico il problema della allocazione dei costi derivanti dai danni provocati da prodotti conformi agli standards di sicurezza non sembra emergere con una sufficiente chiarezza né nell'analisi della casistica giurisprudenziale, né nelle ricostruzioni interpretative, che tendono a polarizzarsi intorno al problema dei danni cagionati dal prodotto difettoso.

La questione è stata individuata con maggiore lucidità nel sistema giuridico statunitense, ove, anche grazie al decisivo apporto fornito dell'analisi economica del diritto ${ }^{38}$, il problema dell'allocazione del residue of unavoidable risk connessi all'utilizzo di prodotti conformi a standards legislativi costituisce da tempo oggetto di una importante elaborazione interpretativa condotta in sede legislativa e giurisprudenziale. Il sistema giuridico statunitense, quindi, costituisce un modello da osservare con estremo interesse in quanto fornisce una significativa conferma riguardo alla necessità di adottare una lettura rigorosamente coordinata delle regole che governano la responsabilità del produttore e di quelle che individuano standards uniformi di sicurezza dei prodotti e consente di risolvere i delicati problemi relativi alla responsabilità del fabbricante per i danni cagionati da prodotti conformi agli standards di sicurezza federali secondo un disegno sistematico che in linea di principio riflette le scelte operate in termini generali dal legislatore. Per questa ragione sembra opportuno far precedere la rilettura critica degli orientamenti giurisprudenziali formatisi nel nostro ordinamento dall'illustrazione delle linee guida che emergono dalla casistica giurisprudenziale statunitense in materia di responsabilità per i danni cagionati dai prodotti conformi agli standards di sicurezza, ma inevitabilmente dannosi.

\footnotetext{
${ }^{38}$ Il decisivo apporto fornito alla elaborazione interpretativa in materia di product liability statunitense da Calabresi, The Cost of Accidents. A Legal and Economic Analysis, New Haven and London, 1970 emerge chiaramente in molti passidel Restatement Third.
}

176 R. Jur. FA7, Fortaleza, v. 13, n. 2, p. 165-189, jul./dez. 2016 


\section{LA RESPONSABILITÀ DEL FABBRICANTE PER IL DANNO DA PRODOTTO CONFORME. LE SOLUZIONI DEL DIRITTO STATUNITENSE}

Nel contesto statunitense la distinzione tra il problema del danno cagionato da un prodotto difettoso e quello del danno derivante da prodotto conforme, ossia non difettoso, ma inevitabilmente dannoso, emerge, anzitutto, dalle regole sistematizzate nei Restatement Second e Third, dedicati alla materia dei Torts. L'elaborazione giurisprudenziale, infatti, aveva condotto nella prima metà degli anni Sessanta a ritenere che il prodotto potesse essere considerato difettoso quando risultava unreasonably dangerous; il che portava ad escludere, in termini speculari, una responsabilità del produttore nel caso in cui si verificassero danni in ragione dell'utilizzo "normale" di un prodotto ragionevolmente sicuro ${ }^{39}$.

Sotto questo profilo già le note illustrative della section 402 A del Restatement Second sottolineavano la rilevante distinzione — poi ribadita dal Restatement Third — tra prodotto difettoso e prodotto sicuro che, inevitabilmente, conserva margini di dannosità "accettabili". Prendendo come esempio le bevande alcoliche, si specifica che è da considerare unreasonably dangerous una bevanda alcolica che contiene sostanze capaci di arrecare danni anche in caso di uso appropriato ${ }^{40}$; al tempo stesso non si dubita che non possa considerarsi unreasonably dangerous, e quindi difettoso, il prodotto alcolico di buona qualità solo perché il suo consumo può provocare gravi danni in caso di uso inappropriato o eccessivo. Del resto, continua il commento illustrativo, questo genere di considerazioni possono essere ripetute anche con riferimento a molteplici tipologie di prodotti tradizionali di uso comune come, ad esempio, il tabacco, il sale, lo zucchero, il burro, di per sé non unreasonably dangerous, ma estremamente dannosi se consumati in modo inappropriato. Le osservazioni appena svolte conducono ad individuare con maggiore precisione la categoria dei c.d. unavoidable unsafe products, ossia a quei prodotti che, anche nell'ambito del loro uso normale, conservano significativi margini di dannosità che allo stato delle conoscenze non è possibile eliminare quantomeno sostenendo costi di fabbricazione ragionevoli. A tale riguardo vengono portati ad esempio i prodotti farmaceutici e, in particolare il caso di alcuni vaccini che, pur comportando il rischio di gravi effetti collaterali, non possono essere considerati difettosi, né unreasonably dangerous. Le stesse automobili, del resto, presentano caratteristiche costruttive che consentono di raggiungere un livello di sicurezza solamente ragionevole, assai lontano da quella assoluta. Proprio con riferimento a quest'ultima tipologia di prodotti le illustrations del Restatement precisano che il livello di sicurezza ragionevole richiesto dall'ordinamento deve essere individuato anche tenendo conto dei costi produttivi. Pertanto, se da un lato può considerarsi irragionevole un livello di sicurezza troppo basso, dall'altro può apparire non appropriato anche il conseguimento di un livello di sicurezza molto elevato o assoluto che comporti costi produttivi eccessivi e limiti irragionevolmente la diffusione di un prodotto circoscrivendo la possibilità di accesso al suo utilizzo ai soli consumatori dotati di un ingente disponibilità economica ${ }^{41}$.

\footnotetext{
${ }^{39}$ Restatement (Second) of Torts, Chapter 14, section 402 A, comment i, p. 353.

${ }^{40} \mathrm{Cfr}$. Restatement (Second) of Torts, Chapter 14, section 402 A, comment i, p. 353.

${ }^{41}$ Sotto questo profilo emerge il problema di contemperare i benefici connessi alla diffusione di determinati prodotti ed i rischi che la stessa diffusione di quei prodotti può comportare in termini di pericoli per l'integrità e la salute delle persone. In proposito il Restatement Third, Torts: Products Liability, Chapter 1, section 2, comment a, p. 16, chiarisce che la società, nel suo complesso, non trae un beneficio dalla diffusione di excessively safe products. In tal senso viene indicato l'esempio di automobili progettate per raggiungere una velocità massima di 20 miglia orarie. Secondo quanto si afferma nel comment a del Restatement Third, Torts: Products Liability, Chapter 1,
} 
Il danno da "prodotto conforme": le soluzioni europee e statunitensi nella prospettiva del Transatlantic Trade and Investment Partnership (T.T.I.P.)

La distinzione tra prodotto difettoso e prodotto conforme, ma comunque dannoso emerge chiaramente nei settori - ormai sempre più numerosi — in cui la definizione dello stato dell'arte è stata trasfusa in specifiche disposizioni di legge o in "standards tecnici" predisposti da agenzie governative (Agencies).

In termini generali, si può affermare che il mancato rispetto degli standards imposti dalla legge indichi la difettosità del prodotto ${ }^{42}$. Sotto questo profilo la section 4 del Restatement Third, intitolata Noncompliance and Compliance with Product Safety Statutes or Regulations, fornisce spunti di estremo interesse ai fini dell'analisi dei rapporti tra regole "preventive" e norme risarcitorie. In essa infatti è chiaramente enunciato il principio secondo cui in relazione alla responsabilità per difetto di progettazione o inadeguata informazione il prodotto si deve considerare difettoso qualora risulti non conforme (noncompliance) alle previsioni sulla sicurezza disposte dalla legge o da regolamenti (Restatement Third, Torts: Products Liability $\S 3$, (a) $)^{43}$.

Se, da un lato, la mancata conformità si risolve automaticamente in un giudizio di difettosità del prodotto, non può affermarsi che, reciprocamente, il prodotto conforme sia di

section 2, infatti, questo livello di sicurezza così elevato comporterebbe costi eccessivi e limiterebbe sensibilmente il livello di utilità sociale dell'automobile. Se ciò accadesse, si determinerebbe, di fatto, l'esclusione dall'utilizzo di questa categoria di beni delle fasce di consumatori economicamente più deboli. Questo fenomeno è stato intuito e profondamente indagato già nei primi anni Settanta dagli studi statunitensi che, attraverso l'analisi economica del diritto, misero in luce il problema di adottare scelte di politica del diritto capaci di assicurare una razionale sopportazione dei costi connessi alla diffusione di attività e prodotti indispensabili ma, al tempo stesso inevitabilmente portatori di fattori di rischio per la salute delle persone (Calabresi, The Cost of Accidents: A Legal and Economic Analysis, New Haven, 1970). Così, nello studio che rappresenta tuttora un imprescindibile punto di riferimento, Guido Calabresi sottolineava che "ogni scelta relativa ad un prodotto, o all'uso di un prodotto, comporta, più o meno implicitamente, una decisione in termini di sicurezza e di costo" (Calabresi, Costo degli incidenti e responsabilità civile. Analisi economico-giuridica, traduzione di De Vita, Varano, Vigoriti, Presentazione di S. Rodotà, Milano, 1975, ristampa inalterata con Presentazione di Al Mureden, Milano, 2015, p. 40). Sotto questo profilo risulta estremamente incisivo l'esempio considerato da Calabresi proprio riguardo alle scelte adottate in materia di sicurezza delle automobili. Egli notava, già negli anni Settanta, che da molto tempo la tecnica consentiva di costruire automobili assolutamente sicure e quindi di ridurre drasticamente gli incidenti a cui conseguono lesioni serie o letali per gli occupanti. La sicurezza, tuttavia, sottolinea Calabresi, "costa" e la scelta di costruire auto che offrano un livello di sicurezza solamente ragionevole, ma assai lontano da quello appena indicato, costituisce un'emblematica dimostrazione di come l'ordinamento adotti scelte di compromesso che consentono di contemperare le esigenze di tutela della vita della salute umana con altre esigenze antagonistiche. In termini più generali, ed ancora più espliciti, lo stesso Calabresi osserva che le lesioni e gli incidenti letali inevitabilmente correlati alla circolazione stradale ed alla diffusione di altri prodotti può essere osservata come "un insignificante peso da pagare per una società in progresso tecnologico che, poiché si è affidata ad un progresso di questo tipo, può curare la difterite, la polmonite e tutta una serie di malattie del passato" (Calabresi, Il dono dello spirito maligno, Milano, 1996, traduzione di C. Rodotà, con Presentazione di Mazzoni, p. 20). Con riferimento all'ordinamento italiano Carnevali, Prevenzione e risarcimento nelle direttive comunitarie sulla sicurezza dei prodotti, cit., p. 15 osserva che « gli standards stabiliti dalle direttive sulla sicurezza rappresentano un equilibrato punto di incontro tra le esigenze dell'industria a programmare e le legittime aspettative degli utenti e consumatori sulla sicurezza d'uso di un prodotto che non può essere assoluta » (sul punto si veda anche Carnevali, voce Nuovi prodotti dannosi, in XXI Secolo, Enc. giur. Treccani, Roma, 2009, p. 347 ss.). Nel contesto europeo considerazioni analoghe riecheggiano in un importante studio della metà degli anni Ottanta (Beck, La società del rischio: verso una seconda modernità, edizione italiana a cura di Privitera, Roma, 2000, pp. 84-92) nel quale, con un'espressione particolarmente incisiva e carica di giudizi decisamente critici, si è messo in luce il cd. "imbroglio dei valori massimi consentiti" proprio per indicare che il riferimento a soglie "accettabili" di esposizione a fattori di rischio finisce per rendere possibile "una razione permanente di avvelenamento collettivo standardizzato".

${ }^{42}$ Il comment e del Restatement Third, Torts: Products Liability section 4, chiarisce che "when a product design is in violation of a safety statute or regulation, there is no necessity to prove an alternative design in order to establish defect. Section 4 makes it clear that a product design that is in violation of safety standards is defective per se".

${ }^{43}$ Il comment e Restatement Third, Torts: Products Liability section 4, p. 121, precisa che occorre che la regola violata sia chiara e che essa imponga l'adozione di determinati standards; dunque ove la regola abbia una valenza facoltativa e nei casi in cui non sia univoca, non è possibile addivenire ad un giudizio di difettosità rispetto al prodotto che non si allinei ad essa. 
per sé sicuro. Il comment e, infatti, chiarisce che il prodotto conforme alle leggi o ai regolamenti (regulations) emanati delle agenzie statali e federali in materia di sicurezza può conservare significativi margini di dannosità e, pertanto, essere considerato difettoso. Ciò consente di affermare che le norme sulla sicurezza dei prodotti - siano esse statali o federali devono essere intese, in linea di massima, come minimum standards ${ }^{44}$. In altri termini, esse stabiliscono solamente una soglia minima di sicurezza (floor) al di sotto della quale il produttore deve considerarsi sicuramente responsabile; il che, tuttavia, non esclude di operare valutazioni circa l'opportunità di adottare livelli di sicurezza più elevati e, in ultima analisi, di affermare la responsabilità del produttore che abbia omesso di adottarli ${ }^{45}$.

Proprio con riferimento ai c.d. dangerous though not defective products, ossia per quei prodotti che, sebbene fabbricati secondo le regole dell'arte, conservano, inevitabilmente, elevati margini di dannosità ${ }^{46}$ è emersa l'esigenza di condurre i giudizi relativi alla responsabilità del produttore sulla base di un rigoroso raffronto tra le caratteristiche del bene da cui è scaturito il danno e quelle prescritte negli standards legislativi che governano la sicurezza di quella particolare tipologia di prodotto.

L'analisi complessiva delle decisioni riferite a diverse tipologie di prodotti evidenzia, in prima approssimazione, una certa disomogeneità per quanto concerne la valenza attribuita alla conformità del prodotto rispetto agli standards di sicurezza sanciti dalle agenzie federali. Nel contesto automobilistico, ad esempio, gli standards federali sulla sicurezza assumono, in linea di principio, la valenza di requisiti minimi (floor). Il loro rispetto è necessario al fine di conseguire l'omologazione da parte della National Highway Traffic Safety Agency (N.H.T.S.A.), ma non costituisce, in linea di massima, una causa di esonero di responsabilità per il produttore; occorre considerare, tuttavia, che questo principio - valido in termini generali - ha subito significative smentite anche in decisioni di rilevante importanza ${ }^{47}$. Anche con riferimento alla responsabilità del produttore di farmaci possono

${ }^{44}$ In questo senso si veda la decisione Feldman v. Lederle Labs., 132 N.J. 339, 625 A.2d 1066 (1993), relativa ad un danno permanente cagionato a seguito dell'utilizzo di un farmaco in età infantile. Il rispetto delle regole federali in materia di istruzioni sull'impiego del farmaco non fu ritenuto sufficiente ad escludere una responsabilità del fabbricante, che avrebbe dovuto adottare precauzioni addizionali ed informare il consumatore anche sotto questo specifico profilo.

${ }^{45}$ Il comment e Restatement Third, Torts: Products Liability section 4, p. 123, chiarisce che questa è la regola seguita nella maggior parte dei precedenti. In linea di principio le regole recenti, che non siano rese obsolete da innovazioni tecnologiche e che siano state formulate a seguito di un procedimento completo, trasparente e supportato da studi scientifici condivisi vengono ampiamente considerate. Così nella decisione Miller v. Lee Apparel CO. 11/8/1994, è stato negato il risarcimento del danno subito da un indumento in poliestere che aveva preso fuoco in una situazione particolarmente critica. L'indumento in questione, infatti, risultava rispettoso degli standards federali in materia di infiammabilità. Occorre rilevare, peraltro, che in dottrina è stato posto in evidenza il problema dei conflitti di interesse e delle pressioni esercitate dalle lobbies sull'attività di roulemaking delle Agencies ed è stata suggerita l'adozione di un approccio interpretativo secondo il quale - ove lo standard legislativo risulti obsoleto o comunque inadeguato a fornire un ragionevole livello di protezione - sarebbe opportuno che la Corte attribuisse un rilevo assai limitato alla compliance defense e potesse considerare il prodotto difettoso anche qualora esso sia conforme agli standards legali (in tal senso v. Johnson, Liberating Progress and the Free Market from the Specter of Tort Liability, 83 Nw. U. L. Rev. 1026, 1048-54 (1989)). Cfr. il comment e Restatement Third, Torts: Products Liability section 4,p. 123.

${ }^{46}$ Il Restatement (Second) of Torts, Chapter 14, section 402 A, comment $k$, p. 353, menziona a tale proposito l'es. dei vaccini, delle bevande alcoliche e dei farmaci.

${ }^{47}$ Nella leading decision Geierv. American Honda Company, 529U.S. 861 (2000), riguardante il danno subito dal conducente a causa della mancata installazione dell'air bag sulla sua auto, la Corte Suprema ha escluso la responsabilità del produttore affermando il principio secondo cui detta responsabilità è esclusa ove sia riscontrabile un'indicazione del legislatore federale che attribuisce agli standards di sicurezza la valenza di "limite massimo" di sicurezza (implied preemption), conseguito il quale non è configurabile un danno ingiusto meritevole di essere risarcito. Tale principio è stato ulteriormente ribadito nella successiva decisione Morgan v. FordMotor Co., No. 34139(W.V.Sup.Jun. 18,2009). 
Il danno da "prodotto conforme": le soluzioni europee e statunitensi nella prospettiva del Transatlantic Trade and Investment Partnership (T.T.I.P.)

essere ripetute considerazioni analoghe. In questo ambito, infatti, sembra prevalere la regola secondo cui il rispetto degli standards sanciti dalla FDA costituisce solamente una soglia minima di sicurezza il cui conseguimento non esime in alcun modo il fabbricante dall'adozione di precauzioni addizionali e dalla responsabilità per i pregiudizi provocati dal prodotto conforme alla disciplina pubblicistica della sicurezza, ma comunque caratterizzato da una persistente attitudine a provocare danni ${ }^{48}$.

Occorre tenere conto, peraltro, che trova significative conferme anche l'assunto secondo cui il rispetto degli standards sanciti dalle agenzie federali può costituire un limite massimo di sicurezza, conseguito il quale non è possibile configurare una responsabilità per i pregiudizi cagionati dal prodotto "inevitabilmente dannoso". Così, dando applicazione a questa regola, è stata esclusa la responsabilità dei produttori di sigarette ${ }^{49}$, di telefoni cellulari ${ }^{50}$ e di

${ }^{48}$ Cfr. la leading decision Wyeth v. Levine, 555 U.S. No. 06-1249, March 4, 2009, concernente un danno cagionato dall'utilizzo di un farmaco non corredato da adeguate informazioni volte a garantire un uso ragionevolmente privo di rischi. In questo caso la tesi secondo cui non avrebbe potuto essere instaurata un'azione di responsabilità per difetto di informazione laddove il produttore avesse fornito le informazioni richieste secondo gli standards previsti dalla stessa F.D.A. non fu accolta dalla Corte che, invece, affermò la responsabilità del fabbricante. Ciò perché nel Food and Drug Cosmetic Act, è prevista una saving clause in ragione della quale la legislazione statale più stringente della regola federale o comunque non in contra sto con essa può essere esclusa dall'effetto della preemption. In tal caso, pertanto, la conformità allo standard federale non risultò sufficiente ad escludere una responsabilità del fabbricante.

${ }^{49}$ Negli Stati Uniti la protezione della salute dei consumatori viene assicurata attraverso previsioni in materia di etichettatura che rimettono alle opzioni della singola persona debitamente informata la decisione di sottoporsi ai rischi inevitabilmente connessi al consumo di tabacco (15 USC § 1331-Congressional Declaration of Policy and Purpose). Così i doveri di informazione dei produttori di tabacco vengono regolati con una articolata previsione che descrive dettagliatamente la tipologia di messaggi e le modalità grafiche ${ }^{50}$ da adottare per rendere immediatamente percepibili ai consumatori i gravi rischi connessi al consumo di sigarette e altri prodotti affini (15 USC $\S$ 1333-Labeling; Requirements; Conspicuous Statement). Tale previsione è correlata da una clausola esplicita di preemption (15 USC $\S 1334$-Preemption). La normativa attuale appare in linea con una scelta dipolitica del diritto già ampiamente consolidata e risalente al Public Health Cigarette Smoking Act del 1969, che rappresentò, ad opinione di alcuni interpreti, un vero e proprio "scudo" contro le azioni di responsabilità intentate nei riguardi dei produttori sulla base di leggi dei singoli stati. L'assunto secondo cui il rispetto dei doveri imposti ai produttori di sigarette dalla legislazione federale costituisce un "limite massimo" conseguito il quale non è configurabile alcuna responsabilità è stato efficacemente confermato dalla leading decision Cipollone v. Liggett Group, Inc., 505 U.S. 504, 530 n. 27 (1997), in cui fu sancito che il Public Health Cigarette Smoking Act del 1969 prevedeva una clausola di preemption esplicita capace di inibire le azioni risarcitorie promosse contro i produttori che si fossero attenuti agli obblighi imposti dalla legge. L'orientamento appena riportato ha trovato ulteriori conferme in tempi più recenti. Così, un'altra importante decisione (Altria Group v. Good, 555 U.S. 70 (2008)) ha confermato che il Congresso ha manifestato l'intenzione di adottare un unico standard federale per quanto concerne le informazioni da fornire ai potenziali consumatori di prodotti derivati dal tabacco ed ha chiaramente inteso attribuire ad esso la valenza di uno standard massimo (15 U. S. C. $§ 1334(b)$. Tale scelta risponde alla fondamentale esigenza di rimuovere ostacoli alla circolazione delle merci tra i diversi stati americani; esigenza che risulterebbe drasticamente compromessa dalla frammentazione delle singole discipline nazionali. L'adozione di una soluzione che non tenga conto delle finalità di armonizzazione che sottendono la legislazione federale, del resto, finirebbe per dare vita ad una espansione incontrollata di azioni legali basate sulla presenza di difetti d'informazione. Per una esaustiva ricostruzione del problema negli Stati Uniti e nell'Unione Europea v. Howells, The Tobacco Challenge. Legal Policy and Consumer Protection, Ashgate, 2011.

${ }^{50}$ Uno dei contesti nei quali è possibile percepire con maggiore chiarezza la funzione della preemption doctrine come limite alla responsabilità civile e comprendere le ragioni poste al suo fondamento è indubbiamente quello delle decisioni relative ai possibili profili di dannosità connessi all'utilizzo intensivo del telefono cellulare. L'assunto secondo cui la responsabilità del produttore deve arrestarsi di fronte al rispetto delle norme tecniche in cui è sintetizzato lo stato dell'arte - già sancito in precedenza dalla decisione Murray v. Motorola, Inc., 982 A.2d 764 (D.C. 2009) - ha trovato la sua conferma più significativa nella decisione Farina v. Nokia,625 F. 3d 97, 2010 U.S. App. Lexis 22.383, 51, Comm. Reg. (P \& F) 955. In questo caso era stata promossa una class action volta ad accertare la pericolosità dei telefoni cellulari nelle condizioni di utilizzo normale previste dagli standards elaborati a livello federale dalla Federal Communication Commission (FCC). Tali standards, infatti, risultavano meno protettivi di quelli previsti dalla legge della Pennsylvania soprattutto laddove non imponevano l'adozione di accorgimenti necessari (in particolare gli auricolari) a ridurre al minimo il rischio di patologie connesse all'emissione di radiofre- 
alcolici $^{51}$ che si erano limitati ad adottare le misure di prevenzione dei danni richieste dagli standards legislativi enon si erano spinti sino aricorre a tutte quelle che sarebbero state necessarie per limitare ulteriormente la dannosità connessa alla diffusione di quei prodotti.

La rassegna della casistica giurisprudenziale fin qui illustrata dimostra che — al di là degli apparenti profili di contraddittorietà — le differenti soluzioni indicate scaturiscono da una lettura rigorosamente coordinata delle discipline federali sulla sicurezza dei prodotti e dei regolamenti emanati dalle Agencies. Esse, quindi, riflettono — in linea di massima - le differenziate scelte di politica del diritto attuate dal legislatore e circostanziate dalle Agencies con riferimento alle molteplici categorie di prodotti dai quali possono scaturire rischi per gli utilizzatori. In altri termini, quindi, emerge una propensione del giudice ad attuare una lettura della tort law rigorosamente rispettosa delle scelte operate dal legislatore e dalle Agencies federali, quindi compendiate nella cosiddetta safety law federale.

Il "percorso predefinito" che il giudice è chiamato a seguire quando si tratta di decidere riguardo alla risarcibilità dei danni cagionati dall'utilizzo di un prodotto si articola,

quenze né la previsione di avvertenze volte ad incentivare un utilizzo responsabile e sicuro. La Corte Suprema, invero, ha escluso profili di responsabilità in capo al produttore che si limiti ad adeguarsi agli standards di sicurezza federali e commercializzi telefoni non dotati dell'auricolare. La decisione assume particolare interesse in quanto nel ribadire il principio della prevalenza degli standards di sicurezza adottati a livello federale rispetto a quelli sanciti dalla legislazione statale - sottolinea significativamente la necessità di individuare nel conseguimento di un livello di "sicurezza ragionevole" un limite alla responsabilità del produttore ed illustra puntualmente le ragioni di ordine generale che giustificano questa scelta di politica del diritto. Infatti, la permanenza di una rete di telecomunicazione capace di coprire tutto il territorio federale, precisa la motivazione, richiede necessariamente un'uniformità federale degli standards di sicurezza. In questa prospettiva gli standards sanciti dall'Agenzia federale che governa il settore delle telecomunicazioni (Federal Communication Commission F.C.C.) debbono essere osservati come il risultato di complesse valutazioni che tengono in considerazione, da un lato, l'esigenza di tutelare la salute e, al tempo stesso, quelle di corretto funzionamento del sistema e dei costi necessari per raggiungere tale obiettivo. Qualora si ammettesse la possibilità di mettere in discussione gli standards federali attribuendo rilievo a valutazioni diverse operate dai singoli legislatori nazionali, o addirittura sancite da letture giurisprudenziali, si finirebbe per infrangere l'unità del sistema. Assoggettare la disciplina della rete di telecomunicazioni estesa su tutto il territorio federale ad una regolamentazione statale frammentaria (patchwork) comporterebbe costi insostenibili. Poiché - continua la motivazione - l'Agenzia federale è indubbiamente nella migliore posizione per bilanciare gli obiettivi della legge federale con le esigenze di tutela della salute e gli standards che essa individua non possono essere messi in discussione (secondguess) né dalla legislazione statale, né, a maggior ragione, da azioni di responsabilità basate su legislazione statale (c.d. preemption doctrine).

${ }^{51}$ I risvolti applicativi della preemption theory emergono anche nell'ambito dei doveri di informazione che gravano sui produttori di bevande alcoliche. Fino agli anni Ottanta, in assenza di un espresso dovere di informare i consumatori circa i rischi per la salute connessi ad un eccessivo consumo di bevande alcoliche, la giurisprudenza aveva fornito soluzioni non univoche riguardo alla responsabilità dei produttori per difetto di informazione. Secondo l'indirizzo prevalente non poteva configurarsi una responsabilità dei produttori di alcool per difetto di informazione in quanto la consapevolezza circa i rischi connessi ad un consumo eccessivo era radicata e diffusa (c.d.common knowledge). D'altra parte, seguendo un approccio maggiormente orientato verso la protezione della salute dei consumatori, alcune corti avevano affermato una responsabilità dei produttori che non avevano informato adeguatamente i consumatori circa i rischi inevitabilmente correlati al consumo eccessivo di bevande alcoliche. La questione è stata risolta legislativamente nel 1988, quando il Congresso ha emanato l'Alcholic Beverage Labelling Act ed ha imposto ai produttori l'indicazione di messaggi che sottolineassero i rischi connessi ad una assunzione eccessiva di bevande alcoliche ed inducessero le persone ad adottare stili di vita corretti ed optare per un "consumo responsabile" ${ }^{44}$. La previsione, contenuta nel 27 U.S.C. $§ 215$, infatti, è accompagnata da una clausola espressa dipreemption (27U.S.C. §216); le dettagliate disposizioniregolamentari(regulations) che specificano secondo quali modalità confezionare le etichette e fornire le informazioni sono compendiate nel Code of Federal Regulations che indica persino quale debba essere il testo da inserire nelle etichette (27 CFR 16.21-Mandatory Label Information $)^{55}$ operando distinzioni a seconda delle diverse tipologie di prodotti alcolici ${ }^{56}$. In definitiva, dopo la definizione legislativa delle informazioni da comunicare ai consumatori di bevande alcoliche, la possibilità di configurare una responsabilità per difetto di informazione è limitata alle ipotesi in cui il produttore non si adegui allo standard indicato dal legislatore; al tempo stesso il rispetto di quello standard assicura al produttore l'esenzione da qualsiasi responsabilità per i danni riconducibili ad un consumo eccessivo di alcool (Owen, Products Liability Law, Thompson West, St. Paul, MN, II ed., 2008,p. 704). 
Il danno da "prodotto conforme": le soluzioni europee e statunitensi nella prospettiva del Transatlantic Trade and Investment Partnership (T.T.I.P.)

anzitutto, nell'individuazione delle regole federali che definiscono gli standards tecnici di sicurezza del prodotto considerato, quindi nell'interpretazione delle norme federali che attribuiscono al rispetto dello standard tecnico la valenza di "limite massimo" di sicurezza conseguito il quale non è configurabile un'ulteriore responsabilità del produttore, oppure quella di "limite minimo" il cui conseguimento è necessario per escludere la difettosità del prodotto, ma non sufficiente per giustificare l'esenzione del fabbricante da obblighi risarcitori. Nel quadro appena delineato la responsabilità del produttore può configurarsi anzitutto nel caso in cui il prodotto dannoso presenti caratteristiche che si pongono al di sotto dei livelli di sicurezza individuati dagli standards federali. Essa può essere affermata anche quando il prodotto dannoso risulti conforme agli standards federali; ciò accade in tutti i contesti nei quali lo standard federale assume la valenza di un limite minimo di sicurezza. Peraltro la responsabilità del produttore per i danni cagionati dal prodotto conforme, ma inevitabilmente dannoso, deve essere esclusa nei contesti in cui — in ragione di una preemption clause esplicita o implicita - lo standard federale assume la funzione di indicare il livello massimo di sicurezza esigibile, conseguito il quale non è possibile configurare la risarcibilità dei danni inevitabilmente connessi alla diffusione di un determinato prodotto.

Le decisioni giurisprudenziali che sanciscono l'irresponsabilità del produttore per i danni cagionati da prodotti conformi, ma "inevitabilmente dannosi" sottolineano l'imprescindibile necessità di limitare l'espansione della responsabilità civile in funzione delle valutazioni operate a livello federale e destinate a riflettere i loro effetti in uno spazio economico e politico "comune".

Questa esigenza, indubbiamente presente anche nel contesto dell'Unione Europea, risulta particolarmente avvertita negli Stati Uniti anche in considerazione dei caratteri peculiari che caratterizzano il sistema della responsabilità civile in quel ordinamento. Sotto tale profilo occorre considerare la presenza di alcuni strumenti che, operando sinergicamente, possono determinare conseguenze economiche estremamente rilevanti a seguito dell'accertamento di una responsabilità del fabbricante per i danni cagionati dai prodotti. Anzitutto in quel contesto la class action può assumere sia una dimensione circoscritta alla singola giurisdizione nazionale, sia la portata più ampia della Multidistrict Litigation (Rule 23 della Federal Rules of Civil Procedure ${ }^{52}$ ). In questo secondo caso è possibile aggregare in un'unica iniziativa giudiziale le posizioni di danneggiati appartenenti a giurisdizioni statali diverse. Anche i c.d. punitive damages possono contribuire, sotto un diverso profilo, a rendere particolarmente rilevanti le conseguenze economiche connesse alla dannosità dei pro$\operatorname{dotti}^{53}$.

\footnotetext{
${ }^{52}$ Sul punto v. Genevieve G. York-Erwin, The choice of law problem(s) in the class action context, in 84 N.Y.U. Law Rev. 1793, 2009, in cui si chiarisce che:'The class action mechanism allows groups of plaintiffs to combine their claims and sue defendants collectively pursuant to Rule 23 of the Federal Rules of Civil Procedure. Certification is the process by which a court determines that a proposed class's claims are suitable for collective treatment under Rule 23 or its state-law equivalent. If certified as a class, all class members and defendants are bound by the results of the collective action".

${ }^{53}$ Sui punitive damages si veda la section 908 del Restatement Second, Torts del 1979. Sull'esigenza di limitarne l'espansione v. Schlueter, Punitive Damages, Lexis Nexis, VI ed., 2010, I, p, 31; Vidmar and Wolfe, Punitive Damages, Annual Review of Law and Social Science, 2009, vol. 5, pp. 179-199. Per una riflessione sul ruolo dei punitive damages nella definizione delle funzioni della responsabilità civile, Calabresi, The Complexity of TortsThe Case of Punitive Damages, Madden (edited by), Exploring Tort Law, New York, 2005, p. 333 e in part. p. 338, ove richiama la concurring opinion espressa nella decisione Ciraolo v. City of N.Y., 216 F.3d 236 (2nd Cir.), nonché la opinion espressa da Posner nella decisione Federal Deposit Insurance Corporation v. W.R. Grace \& Co. 877 F.2d 614; Calabresi, A Broader View of the Cathedral: TheSignificanceofthe Liability Rule, Correcting aMisapprehension,p.7;A.Mitchell Polinsky \& Steven Shavell, Punitive Damages: An Economic Analysis, 111, Harvard Law Rev. 869, 887-96 (1998); Catherine M. Sharkey, Punitive Damages as Societal Damages, 113 Yale Law $J .347,363-72$ (2003). L'interesse degli interpreti italiani per questo strumento e la necessità di osservarlo anche
} 
Nonostante i significativi profili di differenziazione che caratterizzano il sistema statunitense rispetto a quello dell'Unione Europea sembra sicuramente da osservare con interesse il disegno di politica del diritto che, ispirandosi all'obiettivo dell'armonizzazione delle regole in un contesto giuridico comune, attribuisce fondamentale importanza all'esigenza di operare una lettura delle norme in materia di responsabilità civile rigorosamente coordinata con quelle che governano la sicurezza dei prodotti. Esso, infatti, risulta, in ultima analisi, funzionale a far sì che la responsabilità civile del produttore si espanda in armonia con le valutazioni di carattere generale operate dal legislatore con riferimento all'individuazione di livelli di "sicurezza ragionevole" delle attività dei prodotti. Inoltre, l'idea di assumere lo standard federale come criterio fondamentale sulla base del quale valutare la sicurezza del prodotto, costituisce il presupposto affinché si realizzi un'effettiva armonizzazione nella materia della responsabilità del produttore e, più in generale, una razionalizzazione dei giudizi concernenti la risarcibilità dei danni provocati dai prodotti.

Muovendo da queste considerazioni, sembra opportuno sottoporre ad una revisione critica gli orientamenti giurisprudenziali che si sono formati nel nostro ordinamento e che non sembrano riconducibili ad un disegno organico simile a quello appena descritto.

\section{6 (SEGUE) LE INCERTEZZE DELLA GIURISPRUDENZA ITALIANA}

Il problema della responsabilità del fabbricante per i danni cagionati da un prodotto conforme agli standards legislativi in materia di sicurezza non viene delineato e colto con chiarezza nell'analisi della casistica giurisprudenziale italiana. Essa fa emergere, anzitutto, una limitatissima propensione ad operare una lettura sistematica e coordinata delle norme che regolano la responsabilità del produttore e di quelle che definiscono la sicurezza dei prodotti sulla base degli standards legislativi armonizzati, quindi, in definitiva, a valorizzare quell" "anello di congiunzione" tra le due discipline che anche il legislatore europeo considera imprescindibile ${ }^{54}$. Il che rende impossibile individuare regole generali sulla base delle quali poter stabilire con sicurezza, per ogni tipologia di prodotto, se sussista una responsabilità del fabbricante anche qualora i danni cagionati all'utilizzatore scaturiscano da un prodotto del tutto conforme agli standards legislativi.

I profili di criticità ed incertezza nella soluzione del problema della responsabilità del fabbricante per i danni cagionati da prodotti conformi agli standards legislativi emerge in modo differenziato nei diversi precedenti giurisprudenziali.

In prima approssimazione è possibile individuare tre categorie di decisioni. Alcune affrontano il problema della responsabilità del produttore di beni la cui sicurezza è definita da standards legislativi omettendo, inopinatamente, ogni riferimento a questi ultimi e ricavando il carattere difettoso del prodotto sulla base del criterio generico che si riferisce alla sicurezza che il consumatore può "legittimamente attendere" (art. 6, dir. 85/374/ CEE; art.

nella prospettiva dell'analisi economica del diritto è emerso in particolare nei contributi di Busnelli, Deterrenza, responsabilità civile, fatto illecito, dannipunitivi, in Europa e dir. priv., 2009, p. 909; Ponzanelli, I danni punitivi, in Nuova giur. civ. comm.,2008, II, p. 25; P.Pardolesi, Danni punitivi:frustrazione da "vorrei, ma non posso"?, in Riv. crit. dir. priv., 2007, p. 341. La necessità di uno strumento idoneo ad attuare una finalità di deterrenza è stata auspicata con crescente insistenza anche nel contesto del nostro ordinamento. In questo senso Patti, Il risarcimento del danno e il concetto di prevenzione, in Busnelli e Patti, Danno e responsabilità civile, III ed., Torino, 2013, p. 99, osserva che con specifico riferimento alla materia dei prodotti difettosi "la semplice condanna alla riparazione, cioèal pagamento del danno causato, non permette in molti casi il perseguimento delle finalità preventiva, propria della responsabilità civile".

${ }^{54}$ The Blu Guide on the Implementation of EU Product Rules, cit., p. 10. 
Il danno da "prodotto conforme": le soluzioni europee e statunitensi nella prospettiva del Transatlantic Trade and Investment Partnership (T.T.I.P.)

5, d.p.r. 224/1988; 117 c. cons.) nell'uso normale ${ }^{55}$. Un secondo gruppo di pronunce risolve il problema della risarcibilità dei danni cagionati da prodotti la cui sicurezza è regolata da standards legislativi armonizzati seguendo un metodo interpretativo che denota un distacco ancora più netto rispetto al sistema di regole in materia di responsabilità del produttore e sicurezza dei prodotti predisposto dal legislatore dell'Unione Europea e trasposto dal legislatore nazionale nel nostro ordinamento. Così, dando vita a quella che è stata definita una "casistica parallela" "56, un considerevole numero di decisioni di legittimità e di merito hanno risolto il problema della risarcibilità dei danni cagionati da alcune tipologie di prodotti applicando la disciplina dell'art. 2050 c.c. in luogo di quella di derivazione comunitaria che regola la responsabilità del produttore (artt. 114 c. cons. ss. $)^{57}$. Solamente in un limitatissimo numero di precedenti è stata colta la necessità di risolvere il problema della responsabilità del produttore di beni la cui sicurezza è regolata da dettagliati standards legislativi adottando una lettura interpretativa in funzione della quale la difettosità del prodotto viene determinata sulla base di rigoroso riferimento ai suddetti standards ${ }^{58}$. Le indicazioni che possono essere ricavate da questi precedenti testimoniano una non piena consapevolezza e considerazione del problema della responsabilità per i danni cagionati da prodotti conformi agli standards legislativi. Infatti, l'esiguo numero di decisioni, la laconicità delle motivazioni e la sussistenza di evidenti profili di disomogeneità tra le soluzioni adottate con riferimento alle diverse tipologie di prodotti non permettono di estrapolare regole generali idonee ad orientare con sicurezza l'interprete ogniqualvolta si ponga l'esigenza di stabilire se il danno provocato da un prodotto conforme agli standards legislativi possa essere risarcito.

La consapevolezza riguardo alla netta distinzione che corre tra il problema della responsabilità del fabbricante per i danni cagionati da prodotti difettosi e quello della responsabilità per i danni provocati da prodotti conformi agli standards legislativi sembra emergere, invero, solo in alcune decisioni recenti le cui motivazioni fanno trasparire una crescente attenzione riguardo alla peculiarità del problema dei danni cagionati da prodotti conformi agli standards legislativi. Così, la S.C. è giunta ad escludere la responsabilità del fabbricante per un danno causalmente riconducibile all'utilizzo di uno strumento chirurgico affermando che la circostanza che il danno "sia temporalmente conseguito l'utilizzazione del prodotto" non può necessariamente condurre a conseguire la prova della sua difettosità. In altri termini, quindi, "per riconoscere la difettosità di un prodotto non è sufficiente accertare il danno da questo provocato e la sussistenza di un nesso causale" tra l'utilizzo del prodotto e il danno; ciò "non prova indirettamente, di per sé la pericolosità del prodotto in condizioni normali di impiego, ma solo una più indefinita pericolosità del prodotto, di per sé insufficiente per

\footnotetext{
${ }^{55}$ Con riferimento ai danni provocati da una scala difettosa Trib. Milano 31 gennaio 2003, in Resp. civ. e prev., 2003, p. 1151, con nota di Della Bella, Cedimento di scalaestensibileeresponsabilità delproduttore-progettista: lanozione didanneggiatonella disciplina della responsabilità del produttore; in Danno e resp., 2003, p. 634, con nota di Bitetto, Oltre la siepe: la scala del giardiniere e la responsabilità da prodotto difettoso! Relativamente ai danni cagionati da una caffettiera, Trib. Vercelli 7 aprile 2003, in Danno e resp., 2003, p. 1001, con nota di Ponzanelli, Responsabilità oggettiva del produttore e difetto di informazione.

${ }^{56}$ Cabella Pisu, Cittadini e consumatori nel diritto dell'Unione Europea, cit., p. 635.

${ }^{57}$ Relativamente ai danni cagionati dallo scoppio di bombole a gas Cass. 4 giugno 1998, n. 5484, in Studium Juris, 1998, p. 1119; Cass. 19 gennaio 1995, n. 567, in Mass. Giust. civ., 1995, c. 97; più di recente, Cass. 26 luglio 2012, n. 13214, in De jure. Riguardo ai danni provocati da farmaci Trib. Salerno 2 ottobre 2007, in Rass. dir. farmaceutico, 2008, p. 29; Trib. Brescia 31 marzo 2003, in Rass. dir. farmaceutico, 2004, p. 1221.

${ }^{58}$ In materia di danni cagionati da prodotti cosmetici v. Cass., 15 marzo 2007, n. 6007, in Resp. civ. eprev., 2007, II, p. 1587, con nota di Gorgoni, Responsabilità per prodotto difettoso: allaricerca della (prova della) causadeldanno; Cass. 13 dicembre2010, n.25116, in Nuova giur. civ. comm., 2011, I, p. 590, con nota di Klesta Dosi, L'incerta disciplina dei prodotti abbronzanti. Riguardo ai danni provocati dall'utilizzo di un motoveicolo Trib. Pisa 16 marzo 2011, in Resp. civ. e prev., 2011, 10, p. 2108, con nota di Carnevali, Il difetto di progettazione negli autoveicoli; in Danno e resp., 2012, p. 67, con nota di Bitetto, Dal biscotto al pan carrè: il tortuoso percorso della responsabilità da prodotto.
} 
integrare la responsabilità del produttore, in mancanza del concreto accertamento della violazione degli standards minimi di sicurezza richiesti dalla utenza o dalle leggi in materia"59. Il livello di sicurezza prescritto dagli standards legislativi, quindi, costituisce una soglia al di sotto della quale il prodotto può considerarsi difettoso, ma non corrisponde ad un livello di sicurezza assoluto tale da garantire la "più rigorosa innocuità" del prodotto stesso. In definitiva, per poter configurare una responsabilità del produttore per i danni cagionati dai prodotti che egli immette sul mercato non è sufficiente dimostrare la sussistenza di un nesso causale tra l'utilizzo del prodotto ed il verificarsi del danno; occorre, invece, dimostrare la sussistenza di un "prerequisito" della responsabilità del fabbricante e, segnatamente, che il prodotto dal quale il danno è scaturito possa considerarsi difettoso, ossia non conforme agli standards legislativi che ne definiscono le caratteristiche di sicurezza. Del resto, l'assunto secondo cui "non può considerarsi difettoso ogni prodotto che di per sé presenti una qualsiasi attitudine" a cagionare un danno ricorre anche in altri precedenti di legittimità in materia di danni derivanti dall'utilizzo di cosmetici conformi ai requisiti stabiliti dalla 1. n. 713 del $1986^{60}$.

La distinzione tra prodotto difettoso, ossia non conforme ai requisiti di sicurezza legislativamente prescritti e prodotto che, sebbene conforme a questi ultimi, risulti "pericoloso con riferimento all'uso anormale che se ne fa" emerge in modo ancor più nitido in un recente obiter dictum contenuto in una pronuncia di merito in materia di danni da fumo. Proprio valorizzando questa distinzione è stata esclusa la possibilità di dare applicazione alla disciplina in materia di responsabilità del produttore contenuta nel codice del consumo ed affermata la riconducibilità della fattispecie al più rigoroso regime di responsabilità previsto dell'art. 2050 c.c. ${ }^{61}$. Questa soluzione, del resto, può essere considerata una applicazione del principio che la S.C. ${ }^{62}$ aveva già espresso anni addietro, stabilendo che l'attività di produzione di sigarette deve essere considerata attività pericolosa ed assoggettata alla disciplina dell'art. 2050 c.c. ${ }^{63}$.

La responsabilità del fabbricante per i danni cagionati da un prodotto conforme agli standards legislativi è stata affermata anche in una decisione di merito nella quale si trattava di decidere riguardo ai danni subiti dal conducente di un motoveicolo pienamente conforme agli standards di sicurezza europei (ECE) ed americani $(F M V S S)^{64}$; standards che, tuttavia, ad opinione del giudicante costituivano solamente una condizione necessaria al fine di conseguire l'omologazione, ma non sufficiente ad escludere una responsabilità del produttore. Tale responsabilità, peraltro, veniva riaffermata anche sotto il profilo del difetto di informazione, ravvisato nella assenza nel libretto di istruzioni un'avvertenza che indicasse all'utilizzatore l'inidoneità strutturale del veicolo di garantire un'adeguata protezione degli occupanti in caso di incidente a velocità superiori al limite dei $50 \mathrm{~km} / \mathrm{h}$.

L'eterogeneità delle soluzioni che emerge dall'analisi delle poche decisioni nelle quali si è posto ed è stato esplicitamente individuato il problema della responsabilità del

${ }^{59}$ Cass. 29 maggio 2013, n. 13458, in Danno e resp., 2014, con nota di Baldassarre, Responsabilità del produttore: danno risarcibile, onere della prova e logica giuridica.

${ }^{60}$ Cass. 15 marzo 2007 n. 6007, cit.; Cass. 13 dicembre 2010, n. 25116, cit.

${ }^{61}$ Trib. Milano, sez. X, 11 luglio 2014, in Danno e resp., 2014, con nota di Ponzanelli, I danni da fumo: la nuova giurisprudenza milanese.

${ }^{62}$ Cass. 17 dicembre 2009, n. 26516, in Danno e resp., 2011, p. 57, con nota di Monateri, La Cassazione e i danni del fumo: evitare un ennesimo "isolamento" italiano; in Corr. giur., 2010, p. 488, con nota di Ponzanelli, La produzione di sigarette è attività pericolosa; in Danno e resp., 2010, p. 569, con nota di D'Antoni, Il danno da fumo e l'art. 2050 c.c.: "scusate il ritardo", che ha sancito il carattere pericoloso dell'attività diproduzione e commercializzazione di prodotti derivati dal tabacco.

${ }^{63}$ Cass. 17 dicembre 2009, n. 26516, cit.

${ }^{64}$ Trib. Pisa 16 marzo 2011, cit. 
Il danno da "prodotto conforme": le soluzioni europee e statunitensi nella prospettiva del Transatlantic Trade and Investment Partnership (T.T.I.P.)

fabbricante per i danni cagionati da prodotto conforme agli standards legislativi impedisce di individuare un criterio sicuro sulla base del quale prevedere gli esiti di controversie analoghe. Le decisioni in materia di danni derivanti da cosmetici o dall'utilizzo di apparecchiature mediche porterebbe ad escludere la responsabilità del fabbricante per i danni provocati da un prodotto non difettoso, ossia conforme agli standards legislativi di sicurezza. Peraltro le decisioni nelle quali è stata affermata la responsabilità del produttore di sigarette o di un motoveicolo conforme agli standards di sicurezza europei forniscono indicazioni di segno opposto, che, invero, sembrano presentare profili di evidente contrarietà rispetto al disegno dell'armonizzazione della responsabilità del produttore nei diversi paesi che compongono l'Unione Europea ${ }^{65}$.

Nel quadro delineato si inserisce un ulteriore elemento di complessità laddove si consideri che l'esigenza di tutelare coloro che subiscano danni dall'utilizzo di un prodotto conforme a standards legislativi ha indotto alcuni interpreti a valorizzare il principio di precauzione $^{66}$ come criterio di lettura delle norme in materia di responsabilità civile ${ }^{67}$. Più precisamente, si è cercato di attribuire rilevanza al principio di precauzione per giustificare l'applicazione dell'art. 2050 c.c. riguardo al risarcimento dei danni cagionati dall'impiego di farmaci $^{68}$ o provocati dal consumo di determinati alimenti ${ }^{69}$.

In realtà la soluzione interpretativa appena illustrata desta perplessità in quanto giungere a riconoscere al giudice il potere di applicare il regime di responsabilità previsto dall'art. 2050 c.c. ed a "cancellare"70 l'esimente del rischio da sviluppo ${ }^{71}$ solo muovendo dall'enunciazione generica del principio di precauzione significherebbe attribuirgli una competenza che dovrebbe essere riservata al legislatore ed alla P.A. Del resto, l'obiezione ad un utilizzo "diretto" del principio di precauzione da parte del giudice chiamato a decidere della responsabilità civile del produttore risulta avvalorata laddove si consideri che la giurisprudenza comunitaria ha chiarito che detto principio legittima l'adozione di misure restrittive da parte del legislatore e della P.A. in assenza di dati scientifici certi ed è, quindi, eminentemente rivolto alle "autorità amministrative" 72 . Esse sono chiamate a "trasformare" la generica enunciazione del principio di precauzione in misure concrete e circostanziate ${ }^{73}$

${ }^{65}$ In questo senso, Monateri, La Cassazione e i danni del fumo: evitare un ennesimo "isolamento" italiano, cit., p. 57.

${ }^{66}$ Sul principio di precauzione De Leonardis, Il principio di precauzione nell'amministrazione del rischio, Milano, 2005; Del Prato, Il principio di precauzione nel diritto privato: spunti, in Rass. dir. civ., 2009, p. 637; Busnelli, Il principio di precauzione e l'impiego di biotecnologie in agricoltura, in Regole dell'agricoltura, Regole del cibo a cura di Goldoni e Sirsi, Pisa, 2005, pp. 115 ss; Santonastaso, Principio di precauzione e responsabilità di impresa: rischio tecnologico, le attività pericolosa per sua natura. Prime riflessioni in tema di ricerca, in Contr. impr./Eu., 2005, pp. 21-24.

${ }^{67}$ Cfr. il documento predisposto dal Comitato Nazionale di Bioetica (C.N.B.) Il Principio di precauzione: profili bioetica, filosofici, giuridici, pubblicato il 18 giugno 2004 e reperibile all'indirizzo http://www.governo.it/BIOETICA, p. 37.

${ }^{68}$ Sul punto v. Izzo, La precauzione nella responsabilità civile, Padova,2004.

${ }^{69}$ La possibilità di liberarsi dalla responsabilità adducendo la mancanza di conoscenze scientifiche circa la pericolosità del prodotto al momento della sua commercializzazione (cd. rischio da sviluppo) sembra porsi ad opinione di alcuni interpreti che si sono occupati della responsabilità del fabbricante di alimenti (Giardina, La responsabilità civile del produttore di alimenti, in Regole dell'agricoltura, Regole del cibo, cit., p. 101; Galasso, Il principio di precauzione nella disciplina degli OGM, Torino, 2007, p. 60) in contrasto con il principio di precauzione che l'art. 7 del reg. (Ce) n. 178/2002 sancisce in termini generali per tutti i prodotti alimentari.

${ }^{70} \mathrm{~L}$ 'espressione è di Giardina, La responsabilità civile del produttore di alimenti, cit., p.101.

${ }^{71}$ Sui rapporti tra rischio da sviluppo e applicazione dell'art. 2050 c.c. si veda Querci, Il rischio da sviluppo: origini ed evoluzioni nella moderna "società del rischio", in "I 25 anni di products liability", in Danno e resp., 2012, p. 31.

72 Trib. I grado Comunità europee 21 ottobre 2003, n. 392/02.

${ }^{73}$ In definitiva il principio di precauzione costituisce un "nuovo parametro della valutazione della legittimità dell'azione amministrativa" (De Leonardis, Il principio di precauzione nell'amministrazione del rischio, cit., $\mathrm{p}$. 140) e, si potrebbe aggiungere, delle stesse leggi. Esso, quindi, è rivolto in primo luogo al legislatore o all'amministrazione, chiamati ad adottare provvedimenti capaci di contemperare le esigenze di tutela della salute con quelle della libera iniziativa economica e della tutela del mercato. 
dopo aver operato, anche con il supporto di organi scientifici accreditati, una valutazione dei rischi ${ }^{74}$ ed attuato un contemperamento tra le esigenze di tutela della salute indicate dalla scienza ed altri interessi che vengono di volta in volta in considerazione. Le "espressioni circostanziate" del principio di precauzione e le norme in materia di sicurezza dei prodotti possono essere oggetto di un sindacato da parte dei "giudici dei provvedimenti legislativi e regolamentari" 75 , mentre nella prospettiva del giudice civile, chiamato a decidere riguardo al risarcimento dei danni, dovrebbero rappresentare un dato di cui occorre necessariamente prendere atto ed un limite all'espansione della responsabilità civile che non può essere messo in discussione dando applicazione al principio di precauzione ${ }^{76}$.

\section{LA LETTURA COORDINATA DELLE NORME SULLA RESPONSABILITÀ CIVILE E DI QUELLE SULLA SICUREZZA DEI PRODOTTI NEGLI STATI UNITI, NELL'UNIONE EUROPEA...}

Il filo conduttore dell'indagine sviluppata con riferimento al contesto nazionale, alla prospettiva dell'Unione Europea e a quella statunitense può essere riassunto nell'esigenza di individuare criteri affidabili che consentano di stabilire entro quali limiti sia possibile configurare una responsabilità del fabbricante per i danni subiti dall'utilizzo di prodotti che risultino del tutto conformi alle prescrizioni contenute negli standards legislativi. Proprio l'esigenza di creare le condizioni funzionali a realizzare una concorrenza equilibrata tra le imprese che operano e diffondono i loro prodotti in un mercato in cui sono compresi più ordinamenti statali presuppone necessariamente di considerare in termini uniformi il problema dei limiti alla responsabilità del produttore.

Come si è avuto modo di osservare, sia nell'Unione Europea, sia negli Stati Uniti, sono stati predisposti articolati sistemi di regole armonizzate funzionali a garantire che $\mathrm{i}$ prodotti siano "ragionevolmente sicuri". Così negli Stati Uniti la frammentazione nazionale della product liability law viene compensata e superata mediante la predisposizione di regole federali uniformi che definiscono la sicurezza dei prodotti (product safety law) e che rivestono rilievo preminente rispetto alle discipline nazionali (preemption doctrine); nell'Unione Europea l'obiettivo dell'armonizzazione della responsabilità del produttore viene attuato mediante l'operare sinergico della disciplina uniforme delle norme che governano la responsabilità civile (dir. 85/374/CEE) e di quelle che regolano la sicurezza dei prodotti (direttiva 2001/95/CE ed altre direttive "verticali" specificamente dedicate a determinate categorie di prodotti). I complessi apparati di norme che definiscono il livello di "sicurezza ragionevole" che i diversi prodotti debbono conseguire per poter essere commercializzati costituiscono il frutto di delicate soluzioni di compromesso attraverso le quali il legi-

\footnotetext{
${ }^{74}$ Corte giust. Ce 9 settembre 2003, n. 236/01; Trib. I grado Comunità europee 21 ottobre 2003, n. 392/02.

${ }^{75}$ Più precisamente, la Corte giustizia ed il Tribunale di primo grado delle Comunità europee (nel contesto comunitario) e la Corte costituzionale, il T.A.R. ed il Consiglio di Stato (nel contesto interno) verificano che le norme in cui si esprime il principio di precauzione siano giustificate dall'effettiva presenza di rischi che - pur non compiutamente dimostrati - si basino su dati scientifici attendibili. Il raffronto del provvedimento o della legge con i dati scientifici è stato ritenuto decisivo, ad es., per stabilire se la legislazione restrittiva dell'uso dei coloranti alimentari (cfr. il caso Motte; Corte giust., 10 dicembre 1985, C-247/84, Motte, in Racc., 1985, p. 3887), di additivi per pasticceria (cfr. il caso Muller, Corte giust., 6 maggio 1986, C-304/84, Muller) o di quelli utilizzati per la preparazione di una nota Energy drink si basi su rischi sufficientemente dimostrati (Commissione/Francia Corte giust. 5 febbraio 2004, C-24/ 00, Commissione/Francia).

${ }^{76}$ In senso critico, tuttavia, si veda Comandé, La responsabilità civile per danno da prodotto difettoso... assunta con 'precauzione', in Danno e resp., 2013, p. 107 ss.
} 
Il danno da "prodotto conforme": le soluzioni europee e statunitensi nella prospettiva del Transatlantic Trade and Investment Partnership (T.T.I.P.)

slatore individua un punto di equilibrio tra molteplici esigenze potenzialmente antagonistiche. Per poter garantire un'equilibrata concorrenza delle imprese ed una tutela uniforme della salute delle persone in uno spazio economico comune assume un'importanza fondamentale che le regole appena menzionate costituiscano un punto di riferimento condiviso sia quando si tratta di stabilire le condizioni di accesso al mercato, sia quando si pone il problema di sancire la responsabilità del produttore per i danni subiti dagli utilizzatori dei prodotti stessi. Come osservato, quest'esigenza trova riscontro nell'armonizzazione del diritto dell'Unione Europea e nell'uniformità che caratterizza la legislazione federale statunitense in materia di sicurezza dei prodotti. In definitiva, quindi, i complessi sistemi di norme che definiscono gli standards di sicurezza dei prodotti possono essere osservati come strumenti funzionali a garantire l'ordinato sviluppo di un progetto organico nel contesto di un sistema unitariamente concepito. In questo modo la responsabilità civile del produttore si espanderebbe in armonia con le scelte operate in materia di sicurezza dei prodotti; inoltre, in ragione dell'uniformità degli standards, si conseguirebbe l'obiettivo di addivenire ad una uniformità in materia di responsabilità civile in un determinato spazio politico ed economico ${ }^{77}$.

\section{8 ... E NELLA PROSPETTIVA DELL'ARMONIZZAZIONE "TRANSATLANTICA" DEGLI STANDARDS DI SICUREZZA CONSEGUENTE ALL'ATTUAZIONE DEL TRANSATLANTIC TRADE AND INVESTMENT PARTNERSHIP (T.T.I.P.)}

Concentrando l'attenzione sui contesti nei quali la "sicurezza ragionevole" dei prodotti viene definita da norme tecniche funzionali ad attuare esigenze di armonizzazione, si riscontra, sia nell'Unione Europea, sia negli Stati Uniti la necessità che il giudice chiamato a decidere riguardo ai profili risarcitori operi una lettura rigorosamente coordinata delle norme in materia di responsabilità civile e di quelle che governano la sicurezza dei prodotti.

Questo approccio metodologico viene costantemente seguito nelle decisioni statunitensi. In quel contesto il giudice chiamato a decidere riguardo al risarcimento dei danni cagionati dai prodotti opera secondo un "percorso predefinito". Un percorso che, invero, dovrebbe essere adottato anche dal giudice italiano in tutti i casi in cui si tratti di decidere riguardo alla responsabilità del produttore per danni cagionati da un prodotto la cui sicurezza è regolata da norme tecniche armonizzate nelle quali sono contenuti standards uniformi per tutti i paesi dell'Unione Europea. Egli, quindi, dovrebbe anzitutto individuare le regole in ragione delle quali stabilire se il prodotto da cui è scaturito il danno possa considerarsi "ragionevolmente sicuro": qualora il prodotto risulti non rispettoso delle regole che ne definiscono la sicurezza sarà sancita la responsabilità del produttore; nel caso in cui non siano riscontrabili violazioni delle norme che individuano le caratteristiche costruttive del prodotto e degli standards di sicurezza che esso deve rispettare, invece, occorre operare una distinzione basata sulle indicazioni fornite dal legislatore. In particolare la responsabilità del

\footnotetext{
${ }^{77}$ Del resto, la necessità di garantire a tutti coloro che operano in un mercato unico una tendenziale prevedibilità dei giudizi e dei costi inevitabilmente connessi ai danni provocati dalle attività dei prodotti costituisce un elemento fondamentale per la creazione e la sopravvivenza di uno spazio economico unico. In tal senso si vedano le osservazioni di Busnelli e Ponzanelli, La responsabilità del produttore tra legge speciale e Codice civile, in Il danno da prodotti in Italia, Austria, Repubblica federale di Germania, Svizzera, a cura di Patti, Padova, 1990, p. 28, ove si richiama il passo della relazione al progetto di decreto legislativo sulla responsabilità del fabbricante (n. 4) nel quale si rimarca l'esigenza di contenere la responsabilità del produttore entro precisi limiti "idonei a rendere calcolabile il rischio, eliminando le punte del tutto straordinarie ed atipiche”.
} 
produttore per i danni cagionati da un prodotto conforme agli standards potrebbeconfigurarsi qualora le discipline in materia di sicurezza e le norme tecniche armonizzate possano essere considerate funzionali a garantire solamente un livello minimo di sicurezza ${ }^{78}$; in quest'ultimo caso il loro rispetto non consentirebbe di escludere in termini generali la responsabilità del produttore per i danni cagionati da un prodotto conforme alla disciplina sulla sicurezza, ma comunque dannoso. Diversamente, qualora gli standards prescritti dal legislatore assumano la valenza di limiti massimi di sicurezza, si dovrà concludere che non sussiste una responsabilità in capo al fabbricante del prodotto conforme, ma inevitabilmente dannoso ${ }^{79}$.

L'adozione dell'approccio interpretativo indicato risulta indubbiamente funzionale all'attuazione del disegno dell'armonizzazione della responsabilità del fabbricante nei paesi dell'Unione Europea e di quegli obiettivi di efficiente funzionamento del mercato e uniforme tutela delle persone che sono alla base del sistema di regole che governano sicurezza dei prodotti della responsabilità per i danni da essi cagionati. È evidente, pertanto, che una lettura rigorosamente coordinata delle norme in materia di responsabilità del fabbricante $\mathrm{e}$ di quelle concernenti la sicurezza dei prodotti appare, a maggior ragione, imprescindibile nella prospettiva della prossima conclusione del Transatlantic Trade and Investment Partnership (T.T.I.P.).

${ }^{78}$ Cfr. Cass. 31 marzo 2011, n. 7441, in Resp. civ. e prev., 2011, p. 158, con nota di Carnevali, Farmaci difettosi e autorizzazione ministeriale.

${ }^{79}$ Cass. 15 marzo 2007 n. 6007, cit. 